\title{
Parallel Transports in Webs
}

\author{
Christian Fleischhack* \\ Max-Planck-Institut für Mathematik in den Naturwissenschaften \\ Inselstraße 22-26 \\ 04103 Leipzig, Germany \\ Center for Gravitational Physics and Geometry \\ 320 Osmond Lab \\ Penn State University \\ University Park, PA 16802
}

July 17, 2003

\begin{abstract}
For connected reductive linear algebraic structure groups it is proven that every web is holonomically isolated. The possible tuples of parallel transports in a web form a Lie subgroup of the corresponding power of the structure group. This Lie subgroup is explicitly calculated and turns out to be independent of the chosen local trivializations. Moreover, explicit necessary and sufficient criteria for the holonomical independence of webs are derived. The results above can even be sharpened: Given an arbitrary neighbourhood of the base points of a web, then this neighbourhood contains some segments of the web whose parameter intervals coincide, but do not include 0 (that corresponds to the base points of the web), and whose parallel transports already form the same Lie subgroup as those of the full web do.
\end{abstract}

\section{Introduction}

In order to incorporate the full diffeomorphism invariance of general relativity into loop gravity, it is not sufficient to consider only piecewise analytic paths. Instead, at least, piecewise smooth and immersive paths have to be included. This, however, causes a bunch of technical difficulties that are usually related to the fact that two finite graphs need not be contained in a common larger finite graph. This desirable, but not given directedness is necessary to, in particular, make the measure theory well defined. Several attempts have been made to circumvent this problem. First, Baez and Sawin [3] introduced so-called webs. These are certain sets of piecewise smooth immersive paths that are "sufficiently" independent to allow for the definition of the Ashtekar-Lewandowski measure. Later, arbitrarily smooth paths have been shown tractable using hyphs [5, 6]. Nevertheless, there are still several difficulties even in the more restrictive case of smooth webs. One of them is related to the LewandowskiThiemann conjecture [10, which is important for the definition of diffeomorphism-invariant

*e-mail: chfl@mis.mpg.de 
operators in loop quantum gravity. More precisely, since the averaging over the diffeomorphism group is well defined only on a certain subspace of cylindrical functions, it is important that diffeomorphism-invariant operators do indeed preserve that subspace. Lewandowski and Thiemann took the view that this is probably true and argued that to answer that question one should study how parallel transports in portions of webs behave. In the present article we are now going to state several results on this subject. In particular, we will be able to prove that in the case of semisimple structure groups (like $S U(2)$ or $S l(2, \mathbb{C})$ needed for gravity) there are always subpaths of a web that do not run through the base points of the web, but are already holonomically independent, i.e. parallel transports can be assigned to them completely independent of each other. However, one can see [7] that these results are - in contrast to the anticipation expressed in [10] - still not completely sufficient to prove the Lewandowski-Thiemann conjecture. Nevertheless, the methods developed here will be used to prove it in a subsequent article [7].

The present paper goes as follows: After some preliminaries we will introduce the terms "richness" and "splitting". They will be used to encode the relative position of (parts of) webs - do they coincide, are they in a certain sense independent? Next, we will partially exploit an idea, already used in [10], to study under which circumstances groups can be generated by finite products of elements of certain subgroups. Together with some criteria for the holonomical independence of sets of paths, we will finally determine for every web explicitly what parallel transports regular connections may have. In particular, given a web of $n$ paths, one sees that the set of possible parallel transports forms a Lie subgroup of $\mathbf{G}^{n}$ for structure groups $\mathbf{G}$ that are compact Lie. This can be regarded as a proof that is independent from the argumentation in [3]. But, our result is true even if $\mathbf{G}$ is an arbitrary product of semisimple and abelian, possibly noncompact Lie groups. Additionally, we will show that all parallel transports, occurring in a full web, can already be adopted along certain subpaths in a web that are nontrivial in the sense that they do not contain the base points of the web.

\section{Preliminaries}

Let us briefly fix the notations. Throughout the whole paper, $\mathbf{G}$ is some arbitrary group. Starting with Section 5 we assume additionally that $\mathbf{G}$ is a connected Lie group. Fix some arbitrary manifold $M$. Let $\mathcal{P}$ denote the set of all (finite) paths in $M$, i.e. the set of all piecewise smooth and immersive mappings from $[0,1]$ to $M$. $\mathcal{P}$ is a groupoid (after imposing the standard equivalence relation, i.e., saying that reparametrizations and insertions/deletions of retracings are irrelevant) 9, 3. Sometimes we will speak about paths restricted to certain subintervals $I$ of $[0,1]$. By means of some affine map from $I$ to $[0,1]$ we may regard these restrictions naturally as paths again. The set of all smooth connections in some fixed principal fibre bundle $\pi: P \longrightarrow M$ with structure group $\mathbf{G}$ is denoted by $\mathcal{A}$. Given some "ultralocal" trivialization $\iota$ of $P$, we can identify for every path $\gamma \in \mathcal{P}$ and every connection $A \in \mathcal{A}$ the parallel transport w.r.t. to $A$ along $\gamma$ with an element $h_{A}^{\iota}(\gamma) \equiv h_{\gamma}^{\iota}(A)$ of G. Moreover, we define for every finite tuple $\gamma=\left(\gamma_{1}, \ldots, \gamma_{n}\right)$ of paths the set

$$
\mathcal{A}_{\gamma}^{\iota}:=\left\{h_{A}^{\iota}(\gamma) \mid A \in \mathcal{A}\right\} \equiv\left\{\left(h_{A}^{\iota}\left(\gamma_{1}\right), \ldots, h_{A}^{\iota}\left(\gamma_{n}\right)\right) \mid A \in \mathcal{A}\right\} \subseteq \mathbf{G}^{n}
$$

of all possible (tuples of) parallel transports along these paths. Recall that an ultralocal trivialization [8] is simply some collection of trivializations for each single fibre $P_{m}$ in $P$. Note that the assignment of $m \in M$ to that trivialization over $m$ - even locally - need not be smooth. Sometimes, however, we will drop the superscript $\iota$ to simplify notation. Then we assume we are given some arbitrary, but fixed trivialization. It is obvious that $\mathcal{A}_{\gamma}^{\iota}$ is independent of the chosen trivialization, in particular, if it equals the full $\mathbf{G}^{\# \boldsymbol{\gamma}}$. 


\section{Richness and Splittings}

Let $n \in \mathbb{N}_{+}$be some positive integer and let $\mathbf{G}$ be some group.

Definition 3.1 We define

- $\mathcal{V}_{n}$ to be the set of all $n$-tuples with entries equal to 0 or 1 only;

- $\mathbf{G}_{v}:=\left\{\left(g^{v_{1}}, \ldots, g^{v_{n}}\right) \mid g \in \mathbf{G}\right\} \subseteq \mathbf{G}^{n}$ for every $v \in \mathcal{V}_{n}$; and

- $\mathbf{G}_{V}:=\mathbf{G}_{v^{1}} \cdots \mathbf{G}_{v^{k}}$ for every ordered ${ }^{1}$ subset $V=\left\{v^{1}, \ldots, v^{k}\right\} \subseteq \mathcal{V}_{n}$.

We have, e.g., $\mathbf{G}_{(1,0,1,0)}=\{(g, 1, g, 1) \mid g \in \mathbf{G}\}$.

Lemma 3.1 For every $n \in \mathbb{N}_{+}$, every group (Lie group, algebraic group) $\mathbf{G}$ and every $v \in \mathcal{V}_{n}$, the set $\mathbf{G}_{v}$ is a subgroup (Lie subgroup, algebraic subgroup) of $\mathbf{G}^{n}$.

Proof Obviously, $\mathbf{G}_{v}$ is a subgroup of $\mathbf{G}$. If $\mathbf{G}$ is Lie, then it is additionally a submanifold, hence a Lie subgroup of $\mathbf{G}^{n}$. If $\mathbf{G}$ is algebraic, then $\mathbf{G}_{v}$ is Zariski-closed in $\mathbf{G}$, hence an algebraic subgroup.

qed

\subsection{Richness}

Definition 3.2 An ordered subset $V \subseteq \mathcal{V}_{n}$ is called rich iff

1. for all $1 \leq i, j \leq n$ with $i \neq j$ there is an element $v \in V$ with $v_{i} \neq v_{j}$ and

2. for all $1 \leq i \leq n$ there is an element $w \in V$ with $w_{i} \neq 0$.

For instance, let $n=4$. Then $V:=\{(1,1,0,0),(1,0,1,0),(0,1,0,1),(0,0,1,1)\}$ is rich, but $\{(1,1,0,1),(1,0,1,1),(0,1,1,0)\}$ is not, because it fails to fulfill the first richness condition for $i=1$ and $j=4$.

Definition 3.3 Let $n \in \mathbb{N}_{+}$and $K \subset\{1, \ldots, n\}$.

- For every $v \in \mathcal{V}_{n}$ the $K$-restriction $R_{K}(v) \in \mathcal{V}_{n-|K|}$ of $v$ is defined to be the $(n-|K|)$-tuple, that is generated from $v$ by canceling all components of $v$ at the positions listed in $K$.

- For every $V \subseteq \mathcal{V}_{n}$ the $K$-restriction of $V$ is given by

$$
R_{K}(V):=\bigcup_{v \in V}\left\{R_{K}(v)\right\} \text {. }
$$

- For every nonempty $V \subseteq \mathcal{V}_{n}$ the richness deficit $\delta_{V}$ of $V$ is given for $V \neq\{(0, \ldots, 0)\}$ by

and by $\delta_{V}:=n$ otherwise.

$$
\delta_{V}:=\min _{K^{\prime} \subset\{1, \ldots, n\}}\left\{\left|K^{\prime}\right| \in \mathbb{N} \mid R_{K^{\prime}}(V) \text { is rich }\right\}
$$

For example, we have $R_{\{2,4\}}((1,0,1,0))=(1,1)$ and $R_{1}((1,0,1,0))=(0,1,0)$. For convenience, we write $R_{k}$ instead of $R_{\{k\}}$ for $1 \leq k \leq n$. Using the example above of a rich $V$, we get $R_{4}(V)=\{(1,1,0),(1,0,1),(0,1,0),(0,0,1)\}$. Note, finally, that $\delta_{V}$ is well defined. In fact, if $V$ is neither empty nor the zero tuple, it contains at least one nonzero tuple, say $v$ with $v_{i}=1$. There we have $R_{\{1, \ldots, n\} \backslash\{i\}}(V)=\{(1)\}$ which is rich.

\footnotetext{
${ }^{1}$ By an ordered subset of $X$ we mean an arbitrary tuple of elements in $X$ where every element in $X$ occurs at most once as a component of that tuple. However, we will use the standard terminology of sets if misunderstandings seem to be impossible.
} 
Lemma 3.2 Let $V \subseteq \mathcal{V}_{n}$ be nonempty.

1. If $V$ is rich, then $R_{K}(V)$ is rich for all $K \subset\{1, \ldots, n\}$.

2. If $\left(g_{1}, \ldots, g_{k-1}, g_{k+1}, \ldots, g_{n}\right) \in\left[\mathbf{G}_{R_{k}(V)}\right]^{\bullet q}$ for some $q \in \mathbb{N}$, then there is some $g \in \mathbf{G}$ with $\left(g_{1}, \ldots, g_{k-1}, g, g_{k+1}, \ldots, g_{n}\right) \in\left[\mathbf{G}_{V}\right]^{\bullet q}$.

3. $V$ is rich iff $\delta_{V}$ is zero.

Proof Clear.

qed

We remark that $\left[\mathbf{G}_{V}\right]^{\bullet q}$ denotes the $q$-fold multiplication $\mathbf{G}_{V} \cdots \mathbf{G}_{V}$ of $\mathbf{G}_{V}$. In contrast, we use $\mathbf{G}^{n}$ as usual for the $n$-fold direct product $\mathbf{G} \times \cdots \times \mathbf{G}$ of $\mathbf{G}$.

\subsection{Splittings}

Definition 3.4 • A subset $V \subseteq \mathcal{V}_{n}$ is called $n$-splitting iff

1. $\sum_{v \in V} v=(1, \ldots, 1)$ and

2. $(0, \ldots, 0) \notin V$.

- Let $V$ and $V^{\prime}$ be $n$-splittings. $V^{\prime}$ is called refinement of $V$ (shortly: $\left.V^{\prime} \geq V\right)$ iff every $v \in V$ can be written as a sum of elements in $V^{\prime}$.

Lemma 3.3 Let $V$ be some $n$-splitting. Then we have:

- $\mathbf{G}_{V}=\prod_{v \in V} \mathbf{G}_{v}$ independently of the ordering in $V$;

- $\mathbf{G}_{V}$ is a subgroup of $\mathbf{G}^{n}$, hence $\left[\mathbf{G}_{V}\right]^{\bullet 2}=\mathbf{G}_{V}$;

- $\mathbf{G}_{V^{\prime}} \supseteq \mathbf{G}_{V}$ for all $n$-splittings $V^{\prime} \geq V$.

Proof It is easy to see that $\mathbf{G}_{v^{\prime}}$ and $\mathbf{G}_{v^{\prime \prime}}$ commute for all $v^{\prime}, v^{\prime \prime} \in \mathcal{V}_{n}$ with $v^{\prime} v^{\prime \prime}=(0, \ldots, 0)$ where the multiplication is pointwise. Moreover, we then have $\mathbf{G}_{v^{\prime}+v^{\prime \prime}} \subseteq \mathbf{G}_{v^{\prime}} \mathbf{G}_{v^{\prime \prime}}$. Since, as follows directly from the definition, $v^{\prime} v^{\prime \prime}=(0, \ldots, 0)$ for all different elements $v^{\prime}$ and $v^{\prime \prime}$ in an $n$-splitting, $\prod_{v \in V} \mathbf{G}_{v}$ does not depend on the ordering. Now, $\mathbf{G}_{V}=\prod_{v \in V} \mathbf{G}_{v}$ by definition. From $\left(\mathbf{G}_{v}\right)^{-1}=\mathbf{G}_{v}=\mathbf{G}_{v} \mathbf{G}_{v}$ for all $v \in \mathcal{V}_{n}$, we get the group property of $\mathbf{G}_{V}$ and hence $\left[\mathbf{G}_{V}\right]^{\bullet 2}=\mathbf{G}_{V}$. Additionally, if $V^{\prime} \geq V$, then every $v \in V$ is a sum of certain elements in $V^{\prime}$. Consequently, each $\mathbf{G}_{v}$ is contained in $\mathbf{G}_{V^{\prime}}$, whence $\mathbf{G}_{V} \subseteq\left[\mathbf{G}_{V^{\prime}}\right]^{\bullet \# V}=\mathbf{G}_{V^{\prime}}$.

qed

Definition 3.5 Let $n \in \mathbb{N}_{+}$be some positive integer, $S$ be some set and $\vec{s}$ be some $n$-tuple of elements of $S$. Then the splitting $V(\vec{s})$ for $\vec{s}$ is given by

$$
V(\vec{s}):=\left\{v \in \mathcal{V}_{n} \mid v_{i}=1=v_{j} \Longleftrightarrow s_{i}=s_{j}\right\} \backslash\{(0, \ldots, 0)\} \text {. }
$$

For example, the splitting for $\vec{s}=\left(s_{1}, s_{2}, s_{3}, s_{2}\right)$ is $V(\vec{s})=\{(1,0,0,0),(0,1,0,1),(0,0,1,0)\}$.

Lemma 3.4 For every $n, S$ and $\vec{s}$ as given in Definition 3.5 $V(\vec{s})$ is a $n$-splitting.

Proof Let $1 \leq i \leq n$ and $v, v^{\prime} \in V(\vec{s})$ with $v_{i}=v_{i}^{\prime}=1$. Then $v_{j}=1$ iff $s_{i}=s_{j}$. However, the same is true for $v_{j}^{\prime}$. Hence, $v=v^{\prime}$. Since there is at least one $v \in \mathcal{V}_{n}$ with $v_{i}=1$, we get the assertion.

qed

Proposition 3.5 Let $\boldsymbol{f}=\left(f_{1}, \ldots, f_{n}\right)$ be some tuple of continuous functions $f_{i}: X \longrightarrow Y$, where $X$ and $Y$ are topological spaces and $Y$ is assumed Hausdorff.

Then for every $x_{0} \in X$ there is some neighbourhood $U \subseteq X$ of $x_{0}$, such that $V\left(\boldsymbol{f}\left(x_{0}\right)\right) \leq V(\boldsymbol{f}(x))$ for all $x \in U$. 
Proof - By the continuity and the finiteness of $\boldsymbol{f}$, there is for every fixed $x_{0} \in X$ some open $U \subseteq X$ containing $x_{0}$, such that $f_{i}\left(x_{0}\right) \neq f_{j}\left(x_{0}\right)$ implies $f_{i}(x) \neq f_{j}(x)$ for all $x \in U$. Note that $Y$ is Hausdorff.

- Set for every two $n$-splittings $V, V^{\prime}$

$$
\kappa_{V^{\prime}}(v):=\left\{v^{\prime} \in V^{\prime} \mid \exists k: v_{k}=1=v_{k}^{\prime}\right\} .
$$

Then we have $v \leq \sum_{v^{\prime} \in \kappa_{V^{\prime}}(v)} v^{\prime}$ (with $\leq$ defined by $\leq$ on all components). In fact, if $v_{l}=0$, the assertion $v_{l} \leq \sum_{v^{\prime} \in \kappa_{V^{\prime}}(v)} v_{l}^{\prime}$ is trivial. On the other hand, for $v_{l}=1$ there is some $\widehat{v}^{\prime} \in V^{\prime}$ with $\widehat{v}_{l}^{\prime}=1$, i.e. $\widehat{v}^{\prime} \in \kappa_{V^{\prime}}(v)$, hence $v_{l}=1 \leq \sum_{v^{\prime} \in \kappa_{V^{\prime}}(v)} v_{l}^{\prime}$.

- Observe now, that $\kappa_{V(\boldsymbol{f}(x))}(v) \cap \kappa_{V(\boldsymbol{f}(x))}(\widehat{v}) \neq \varnothing$ with $x \in U$ and $v, \widehat{v} \in V\left(\boldsymbol{f}\left(x_{0}\right)\right)$ implies $v=\widehat{v}$. In fact, it implies the existence of some $v^{\prime}$ in that intersection and some $k, l$ with $v_{k}^{\prime}=1=v_{k}$ and $v_{l}^{\prime}=1=\widehat{v}_{l}$. Consequently, $v_{k}^{\prime}=1=v_{l}^{\prime}$, hence $f_{k}(x)=f_{l}(x)$, thus $f_{k}\left(x_{0}\right)=f_{l}\left(x_{0}\right)$ by $x \in U$. Therefore, $v_{k}=1=v_{l}$ and $\widehat{v}_{k}=1=\widehat{v}_{l}$ by $v, \widehat{v} \in V\left(\boldsymbol{f}\left(x_{0}\right)\right)$. This implies $v=\widehat{v}$.

- Altogether we get for every $x \in U$

$$
\begin{array}{rlr}
(1, \ldots, 1) & =\sum_{v \in V\left(\boldsymbol{f}\left(x_{0}\right)\right)} v & \text { (Splitting property) } \\
& \leq \sum_{v \in V\left(\boldsymbol{f}\left(x_{0}\right)\right)} \sum_{v^{\prime} \in \kappa_{V(\boldsymbol{f}(x))}(v)} v^{\prime} & \left(v \leq \sum_{v^{\prime} \in \kappa_{V(\boldsymbol{f}(x))}(v)} v^{\prime}\right) \\
& \left.\leq \sum_{v^{\prime} \in V(\boldsymbol{f}(x))} v^{\prime} \text { (Disjointness of the sets } \kappa_{V(\boldsymbol{f}(x))}(v)\right) \\
& =(1, \ldots, 1) & \text { (Splitting property) }
\end{array}
$$

and, therefore, $v=\sum_{v^{\prime} \in \kappa_{V(\boldsymbol{f}(x))}(v)} v^{\prime}$ with $\kappa_{V(\boldsymbol{f}(x))}(v) \subseteq V(\boldsymbol{f}(x))$ by definition for every $v \in V\left(\boldsymbol{f}\left(x_{0}\right)\right)$.

qed

\section{Generation of Subgroups}

Even if some smooth paths are independent, i.e. they are webs or hyphs, two or more of them may share full segments. Consequently, the parallel transports along these segments are identical. Using the terminology of the previous section, we see that these parallel transports have now values in some subset $\mathbf{G}_{V}$ of $\mathbf{G}^{n}$, where $V$ encodes which segments coincide. In order to prepare the study of the behaviour of the parallel transports along the full paths, we will now present some results on products of sets of type $\mathbf{G}_{V}$.

\subsection{Semisimple Groups}

Before stating the first theorem of this paper, let us recall the definition of the commutator length [4].

Definition 4.1 Let $\mathbf{G}$ be a group that coincides with its commutator subgroup.

- The commutator length $\mathrm{cl}_{\mathbf{G}}(g)$ of an element $g \in \mathbf{G}$ is defined to be the minimal number of commutators in $\mathbf{G}$ whose product equals $g$.

- The commutator length $\mathrm{cl}(\mathbf{G})$ of $\mathbf{G}$ is defined to be $\sup _{g \in \mathbf{G}} \operatorname{cl}_{\mathbf{G}}(g)$.

We remark that $\operatorname{cl}(\mathbf{G})=1$ for all connected semisimple Lie groups that are complex or compact [4. This means, every element in $\mathbf{G}$ can then be written as a commutator of two elements in $\mathbf{G}$.

Theorem 4.1 Let $\mathbf{G}$ be a group that equals its commutator subgroup and let $n$ be some positive integer. Then we have $\mathbf{G}^{n}=\bigcup_{q \in \mathbb{N}}\left[\mathbf{G}_{V}\right]^{\bullet q}$ for any rich ordered subset $V$ of $\mathcal{V}_{n}$.

If, moreover, $\mathbf{G}$ has finite commutator length, then there is a positive integer $q(n)$ such that $\left[\mathbf{G}_{V}\right]^{\bullet q(n)}=\mathbf{G}^{n}$ for any rich ordered subset $V$ of $\mathcal{V}_{n}$. 
Note that $q(n)$ does not depend on the ordering or the number of elements in $V$.

The following proof will exploit an idea presented in [10] for Lie algebras, but now we transfer it to the level of abstract groups.

Proof Let us first prove $\mathbf{G}^{n}=\bigcup_{q \in \mathbb{N}}\left[\mathbf{G}_{V}\right]^{\bullet q}$.

- The case $n=1$ is trivial.

- Let us consider the case $n=2$. By the first richness condition, $V$ has to contain at least one of the elements $(0,1)$ and $(1,0)$. W.l.o.g. we have $(0,1) \in V$. Then by the second richness condition, $(1,0)$ or $(1,1)$ is in $V$. However, we see immediately that both for $i$ equal 0 and 1 we have $\mathbf{G}^{2}=\mathbf{G}_{(0,1)} \mathbf{G}_{(1, i)} \subseteq \mathbf{G}_{V} \subseteq \mathbf{G}^{2}$ if we assume that $(0,1)$ occurs in $V$ before $(1, i)$ does. Completely analogously we have $\mathbf{G}^{2}=\mathbf{G}_{(1, i)} \mathbf{G}_{(0,1)}=\mathbf{G}_{V}$ in the opposite case. Hence, the assertion follows from $\mathbf{G}^{2}=\mathbf{G}_{V} \subseteq \bigcup_{q \in \mathbb{N}}\left[\mathbf{G}_{V}\right]^{\bullet q} \subseteq \mathbf{G}^{2}$.

- We proceed by induction. Assume we have proven the assertion for a certain $n \geq 2$. Let $\left(g_{1}, \ldots, g_{n}, g_{n+1}\right)$ be some element in $\mathbf{G}^{n+1}$.

First, we generate the components 1 to $n$. For this, let $W$ be the $(n+1)$-restriction $R_{n+1}(V)$ of $V$. By Lemma 3.2 $W$ is rich, hence - by induction hypothesis - we have $\left(g_{1}, \ldots, g_{n}\right) \in\left[\mathbf{G}_{W}\right]^{\bullet q}$ for some $q$. Again by Lemma 3.2 there is some $g \in \mathbf{G}$ with $\left(g_{1}, \ldots, g_{n}, g\right) \in\left[\mathbf{G}_{V}\right]^{\bullet q}$.

Second, we generate the last component. Since $\mathbf{G}$ equals its own commutator subgroup, we have some finite number $S$ and finitely many $g_{s}^{\prime}, g_{s}^{\prime \prime} \in \mathbf{G}$ with

$$
\prod_{s=1}^{S} g_{s}^{\prime} g_{s}^{\prime \prime}\left(g_{s}^{\prime}\right)^{-1}\left(g_{s}^{\prime \prime}\right)^{-1}=g^{-1} g_{n+1} .
$$

Moreover, as shown above, there are certain $g_{1, s}^{\prime}$ and $g_{2, s}^{\prime \prime}$ in $\mathbf{G}$, such that

$$
\left(g_{1, s}^{\prime}, 1,1, \ldots, 1, g_{s}^{\prime}\right) \in\left[\mathbf{G}_{V}\right]^{\bullet q^{\prime}} \quad \text { and } \quad\left(1, g_{2, s}^{\prime \prime}, 1, \ldots, 1, g_{s}^{\prime \prime}\right) \in\left[\mathbf{G}_{V}\right]^{\bullet q^{\prime \prime}}
$$

for some $q^{\prime}, q^{\prime \prime}$.

Finally, we get

$$
\begin{aligned}
\left(g_{1}, \ldots, g_{n}, g_{n+1}\right)= & \left(g_{1}, \ldots, g_{n}, g\right)\left(1, \ldots, 1, g^{-1} g_{n+1}\right) \\
= & \left(g_{1}, \ldots, g_{n}, g\right) \cdot \\
& \prod_{s=1}^{S}\left(\left(g_{1, s}^{\prime}, 1,1, \ldots, 1, g_{s}^{\prime}\right)\left(1, g_{2, s}^{\prime \prime}, 1, \ldots, 1, g_{s}^{\prime \prime}\right) \cdot\right. \\
& \left.\left(g_{1, s}^{\prime}, 1,1, \ldots, 1, g_{s}^{\prime}\right)^{-1}\left(1, g_{2, s}^{\prime \prime}, 1, \ldots, 1, g_{s}^{\prime \prime}\right)^{-1}\right) \\
\in & {\left[\mathbf{G}_{V}\right]^{\bullet\left(q+2\left(q^{\prime}+q^{\prime \prime}\right) S\right)} . }
\end{aligned}
$$

Consequently, $\mathbf{G}^{n+1} \subseteq \bigcup_{q \in \mathbb{N}}\left[\mathbf{G}_{V}\right]^{\bullet q}$. The opposite inclusion is trivial.

If, additionally, $\mathbf{G}$ has finite commutator length, the proof is analogous. Just observe that then we may choose $q, q^{\prime}, q^{\prime \prime} \leq q(n)$ and $S \leq \operatorname{cl}(\mathbf{G})$ in the induction step. Setting $q(1):=1, q(2):=1$ and $q(n+1):=(1+4 \operatorname{cl}(\overline{\mathbf{G}})) q(n)$, we get $\mathbf{G}^{n+1}=\left[\mathbf{G}_{V}\right]^{\bullet q(n+1)}$.

qed

Directly from the proof we get a (very weak) upper bound for $q(n)$.

Corollary 4.2 We can choose $q(n) \leq(1+4 \operatorname{cl}(\mathbf{G}))^{n-2}$ for $n \geq 2$, provided $\mathbf{G}$ has finite commutator length.

Moreover, we have 
Corollary 4.3 With the assumptions of Theorem 4.1 we have for every nonempty ordered subset $V$ of $\mathcal{V}_{n}$ and for every $\mathbf{G}$ having finite commutator length:

- $\left[\mathbf{G}_{V}\right]^{\bullet q\left(n-\delta_{V}\right)}$ is a subgroup of $\mathbf{G}^{n}$ isomorphic to $\mathbf{G}^{n-\delta_{V}}$;

- $\left[\mathbf{G}_{V}\right]^{\bullet} q\left(n-\delta_{V}\right)$ equals $\mathbf{G}^{n}$ iff $V$ is rich or $\mathbf{G}$ is trivial;

- $\left[\mathbf{G}_{V}\right]^{\bullet q}=\left[\mathbf{G}_{V}\right]^{\bullet q\left(n-\delta_{V}\right)}$ for all $q \geq q\left(n-\delta_{V}\right)$.

Proof We may assume $V \neq\{(0, \ldots, 0)\}$. Otherwise, the statements are obvious setting $q(0):=1$.

The basic idea is to extract from $V$ the independent and nontrivial components. For this, we divide the set of indices into equivalence classes, whereas $i$ and $j$ are said to be equivalent iff for every element of $V$ its $i$ - and $j$-component coincide. Now, we define for every $v \in V$ a tuple $w$ just by dropping all the components that are 0 for all $v \in V$ or that correspond to indices that are not minimal in their equivalence class. By construction, the set $W$ of all $w$ given this way is rich and the number of components the elements of $W$ have, is $n-\delta_{V}=: n^{\prime} .^{2}$

By Theorem 4.1, we have $\left[\mathbf{G}_{W}\right]^{\bullet q\left(n^{\prime}\right)}=\mathbf{G}^{n^{\prime}}$. By construction, we see that $\left[\mathbf{G}_{V}\right]^{\bullet q\left(n^{\prime}\right)}$ consists precisely of all elements in $\mathbf{G}^{n}$ that are constant on the equivalence classes above or that are the identity if they belong to the equivalence class built by the components being 0 for all $v \in V$. Thus, $\left[\mathbf{G}_{V}\right]^{\bullet q\left(n^{\prime}\right)}$ is indeed a subgroup of $\mathbf{G}^{n}$.

By the group property of $\left[\mathbf{G}_{V}\right]^{\bullet q\left(n^{\prime}\right)}$, we have the second inclusion relation in $\left[\mathbf{G}_{V}\right]^{\bullet q\left(n^{\prime}\right)} \subseteq\left[\mathbf{G}_{V}\right]^{\bullet q}=\left[\mathbf{G}_{V}\right]^{\bullet q\left(n^{\prime}\right)}\left[\mathbf{G}_{V}\right]^{\bullet\left(q-q\left(n^{\prime}\right)\right)} \subseteq\left[\mathbf{G}_{V}\right]^{\bullet q\left(n^{\prime}\right)}$ for all $q \geq q\left(n^{\prime}\right)$. Finally, we see from the construction of $W$ that $\left[\mathbf{G}_{V}\right]^{\bullet q\left(n^{\prime}\right)} \cong\left[\mathbf{G}_{W}\right]^{\bullet q\left(n^{\prime}\right)}=\mathbf{G}^{n^{\prime}}$.

The statement on the equality of $\left[\mathbf{G}_{V}\right]^{\bullet q\left(n-\delta_{V}\right)}$ and $\mathbf{G}^{n}$ is clear now.

qed

Corollary 4.4 The statements of Corollary 4.3 are also true both in the category of Lie groups and that of algebraic groups.

Proof By the construction in the corollary above, we see immediately that $\left[\mathbf{G}_{V}\right]^{\bullet q\left(n^{\prime}\right)}$ is a submanifold of $\mathbf{G}^{n}$ for Lie groups and is closed in $\mathbf{G}^{n}$ for algebraic groups. qed

\subsection{Abelian Groups}

Theorem 4.1 is no longer true if we drop, e.g., the "semisimplicity" condition. For connected and compact $\mathbf{G}$ it can be shown that for

$$
V:=\{(1,1,0,0),(1,0,1,0),(0,1,0,1),(0,0,1,1)\}
$$

we have $\left[\mathbf{G}_{V}\right]^{\bullet q}=\mathbf{G}^{4}$ iff $\mathbf{G}$ is semisimple. (The idea for the proof can be already found in [6, 8].) This example (see Figure 1] on page 13]) corresponds to the web introduced by Baez and Sawin 2] that has been used widely to discuss problems arising in the theory of webs.

However, at least for abelian groups we have a rather explicit description of $\mathbf{G}_{V}$ :

Proposition 4.5 Let $\mathbf{G}$ be some abelian group and let $n$ be some positive integer. Then $\mathbf{G}_{V}$ is a subgroup of $\mathbf{G}^{n}$ for all nonempty ordered subsets $V$ of $\mathcal{V}_{n}$ and we have

$$
\mathbf{G}_{V}=\mathbf{G} \otimes \operatorname{span}_{\mathbb{Z}}(V)=\mathbf{G}^{\operatorname{span}_{\mathbb{Z}}(V)} .
$$

This statement is also true in the category of real and complex Lie groups.

Proof This is a simple consequence of the fact that abelian groups are just $\mathbb{Z}$-modules.

qed

\footnotetext{
${ }^{2}$ On the one hand, $\delta_{V} \leq n-n^{\prime}$ by richness of $W$. On the other hand, it is easy to see that every restriction $W^{\prime}$ of $V$ having elements with more components than those in $W$, is not rich, hence $\delta_{V} \geq n-n^{\prime}$. In fact, it has to contain still equivalent, but different components or components that are always zero.
} 
Note, however, that the equality of $\mathbf{G}_{V}$ and $\mathbf{G}^{n}$ does not only depend on $V$, but also on $\mathbf{G}$. In fact, consider the example $n=4$ and

$$
V=\{(1,1,0,0),(1,0,1,0),(1,0,0,1),(0,1,1,0),(0,1,0,1),(0,0,1,1)\} .
$$

One checks immediately that

$$
\operatorname{span}_{\mathbb{Z}}(V)=\left\{\left(z_{1}, z_{2}, z_{3}, z_{4}\right) \in \mathbb{Z}^{4} \mid z_{1}+z_{2}+z_{3}+z_{4} \equiv 0 \bmod 2\right\} .
$$

Consequently, we have $\left(\mathbb{Z}_{2}\right)_{V} \subset\left(\mathbb{Z}_{2}\right)^{4}$, but $\left(\mathbb{Z}_{3}\right)_{V}=\left(\mathbb{Z}_{3}\right)^{4}$. However, given a Lie group over $\mathbb{R}$ (or $\mathbb{C}$ ) the situation is much nicer because of

Corollary 4.6 For every positive integer $n$ and every ordered subset $V$ of $\mathcal{V}_{n}$ we have

$$
\begin{aligned}
\mathbb{R}_{V} & =\operatorname{span}_{\mathbb{R}} V \\
(U(1))_{V} & =\left(\operatorname{span}_{\mathbb{R}} V\right) / \mathbb{Z}^{n} .
\end{aligned}
$$

In particular, we have $\mathbf{G}_{V}=\mathbf{G}^{n}$ iff $\operatorname{span}_{\mathbb{R}} V=\mathbb{R}^{n}$ for $\mathbf{G}=\mathbb{R}, U(1)$.

For the example $V=\{(1,1,0,0),(1,0,1,0),(0,1,0,1),(0,0,1,1)\}$ from the beginning of this subsection and $\mathbf{G}=\mathbb{R}$, we have $\operatorname{span}_{\mathbb{R}} V=\left\{\left(x_{1}, x_{2}, x_{3}, x_{4}\right) \mid x_{1}-x_{2}-x_{3}+x_{4}=0\right\} \subseteq \mathbb{R}^{4}$. In particular, $\operatorname{dim} \mathbf{G}_{V}=3$, but $n=4$.

\subsection{More General Groups}

If we would restrict ourselves to the case of (connected) compact Lie groups, we can always write $\mathbf{G}$ as $\left(\mathbf{G}_{\mathrm{ss}} \times U(1)^{r}\right) / \mathbf{N}$, where $\mathbf{G}_{\mathrm{ss}}$ is some semisimple compact Lie group, $r$ is some natural number and $\mathbf{N}$ is some discrete central subgroup of $\mathbf{G}_{\mathrm{ss}} \times U(1)^{r}$. We know already the properties of $\mathbf{G}_{V}$ for the semisimple and the $U(1)$ case. Therefore it is natural to investigate, how the subsets generated by $V$ in a product group are related to the corresponding subsets in the single groups and for the factorized version as well. This question will be answered by the following propositions. However, before we can state them, we have to introduce some (sloppy) notation. For $i=1, \ldots, k$ let $\mathbf{G}_{i}$ be some group and $U_{i}$ be some subset of $\left(\mathbf{G}_{i}\right)^{n}$ with a certain fixed $n$. Then we can naturally identify

$$
U_{1} \times \cdots \times U_{k} \equiv\left\{\left(\left(g_{11}, \ldots, g_{1 n}\right), \ldots,\left(g_{k 1}, \ldots, g_{k n}\right)\right)\right\} \subseteq\left(\mathbf{G}_{1}\right)^{n} \times \ldots \times\left(\mathbf{G}_{k}\right)^{n}
$$

with the subset

$$
\left\{\left(\left(g_{11}, \ldots, g_{k 1}\right), \ldots,\left(g_{1 n}, \ldots, g_{k n}\right)\right)\right\}
$$

of $\left(\mathbf{G}_{1} \times \cdots \times \mathbf{G}_{k}\right)^{n}$. Somewhat sloppily we denote this subset also by $U_{1} \times \cdots \times U_{k}$. One sees immediately that properties being a subgroup, being a submanifold etc. are invariant under this identification - its just an isomorphism. Using this we have

Proposition 4.7 Let $\mathbf{G}_{1}, \ldots, \mathbf{G}_{k}$ be some groups, $n$ be a positive integer and $V$ be some ordered subset of $\mathcal{V}_{n}$.

Then we have $\left(\mathbf{G}_{1} \times \cdots \times \mathbf{G}_{k}\right)_{V}=\left(\mathbf{G}_{1}\right)_{V} \times \cdots \times\left(\mathbf{G}_{k}\right)_{V}$.

Proof This is trivial, since the index $V$ just indicates a certain product of elements without mixing any components.

qed

Proposition 4.8 Let $\mathbf{G}$ be a group and $\mathbf{N}$ be a normal subgroup of $\mathbf{G}$. Moreover, let $n$ be some positive integer and $V$ be some ordered subset of $\mathcal{V}_{n}$.

Then $(\mathbf{G} / \mathbf{N})_{V}=\mathbf{G}_{V} / \mathbf{N}^{n}$, which is a subgroup of $(\mathbf{G} / \mathbf{N})^{n} \cong \mathbf{G}^{n} / \mathbf{N}^{n}$.

Proof Clear.

qed

Altogether we have 
Theorem 4.9 Let $\mathbf{G}_{\mathrm{ss}}$ be some group that equals its commutator subgroup and has finite commutator length. Let $\mathbf{G}_{\mathrm{ab}}$ be some abelian group. Moreover, let $\mathbf{N}$ be some normal subgroup of $\mathbf{G}_{\mathrm{ss}} \times \mathbf{G}_{\mathrm{ab}}$. Define $\mathbf{G}:=\left(\mathbf{G}_{\mathrm{ss}} \times \mathbf{G}_{\mathrm{ab}}\right) / \mathbf{N}$.

Then there is a function $q: \mathbb{N} \longrightarrow \mathbb{N}_{+}$, such that $\left[\mathbf{G}_{V}\right]^{\bullet q\left(n-\delta_{V}\right)}$ is a subgroup of $\mathbf{G}^{n}$ for any nonempty ordered subset $V$ of $\mathcal{V}_{n}$ and for every positive integer $n$. For rich $V$ we even have

$$
\left[\mathbf{G}_{V}\right]^{\bullet q(n)}=\left(\left(\mathbf{G}_{\mathrm{ss}}\right)^{n} \times\left(\mathbf{G}_{\mathrm{ab}}\right)_{V}\right) / \mathbf{N}^{n} .
$$

Proof Choose $q(n)$ according to Theorem 4.1 and Corollary 4.3. By the assertions above, we know that

$$
\left[\mathbf{G}_{V}\right]^{\bullet q\left(n-\delta_{V}\right)}=\left(\left[\left(\mathbf{G}_{\mathrm{SS}}\right)_{V}\right]^{\bullet q\left(n-\delta_{V}\right)} \times\left[\left(\mathbf{G}_{\mathrm{ab}}\right)_{V}\right]^{\bullet q\left(n-\delta_{V}\right)}\right) / \mathbf{N}^{n}
$$

which is a subgroup of $\mathbf{G}^{n}$. Additionally we used the group property of $\left(\mathbf{G}_{\mathrm{ab}}\right)_{V}$ to get $\left[\left(\mathbf{G}_{\mathrm{ab}}\right)_{V}\right]^{\bullet q(n)}=\left(\mathbf{G}_{\mathrm{ab}}\right)_{V}$. Moreover, the assertion for rich $V$ follows directly from Theorem 4.1 .

qed

Corollary 4.10 The statements of Proposition 4.7 Proposition 4.8 and Theorem 4.9 (both for discrete $\mathbf{N}$ ) are true in the category of $\mathbb{K}$-Lie groups as well. Here, $\mathbb{K}$ is $\mathbb{R}$ or $\mathbb{C}$. If, moreover, $\mathbf{G}_{\mathrm{ab}}$ is connected, then we have

$$
\begin{aligned}
& \operatorname{codim}_{\mathbf{G}^{n}}\left[\mathbf{G}_{V}\right]^{\bullet q\left(n-\delta_{V}\right)}=\delta_{V} \cdot \operatorname{dim} \mathbf{G}_{\mathrm{SS}}+\operatorname{codim}_{\mathbb{R}^{n}} \operatorname{span}_{\mathbb{R}} V \cdot \operatorname{dim} \mathbf{G}_{\mathrm{ab}} \\
& =\delta_{V} \cdot \operatorname{dim} \mathbf{G}_{\mathrm{ss}}+\left(n-\operatorname{dim} \operatorname{span}_{\mathbb{R}} V\right) \cdot \operatorname{dim} \mathbf{G}_{\mathrm{ab}}
\end{aligned}
$$

for all nonempty subsets $V$ of $\mathcal{V}_{n}$.

Proof The transferability of the assertions above to the category of Lie groups is clear. (Note that the discreteness of $\mathbf{N}$ guarantees that the groups $\mathbf{G}^{n}$ and $\mathbf{G}^{n} / \mathbf{N}^{n}$ are locally diffeomorphic.) The codimension formula again is a consequence of the preceding statements. Observe that every connected abelian Lie group over $\mathbb{K}$ is isomorphic to some $\mathbb{R}^{r} \times U(1)^{s}$. Here, of course, $r+s$ is even for $\mathbb{K}=\mathbb{C}$, such that $\mathbb{R}^{r} \times U(1)^{s}$ can be regarded naturally as a complex Lie group.

qed

\subsection{Application to Reductive Groups}

Proposition 4.11 For every positive integer $n$, every nonempty subset $V$ of $\mathcal{V}_{n}$ and every connected reductive linear algebraic group $\mathbf{G}$ over $\mathbb{R}$ or $\mathbb{C}$, the smallest subgroup generated by $\mathbf{G}_{V}$ is a Lie subgroup of $\mathbf{G}^{n}$. This smallest subgroup is given by $\left[\mathbf{G}_{V}\right]^{\bullet q}$ with $q$ being any integer $q \geq q\left(n-\delta_{V}\right)$. It equals $\mathbf{G}^{n}$ iff $V$ is a generating system for $\mathbb{R}^{n}$, or $\mathbf{G}$ is semisimple with rich $V$.

Note that every (real or complex) linear algebraic group has also a Lie group structure. In what follows, we will always assume to use this Lie structure when speaking about linear algebraic groups. Therefore, we may consider, e.g., Lie subgroups of linear algebraic groups, as we did in the proposition above.

Proof - Recall that every connected reductive linear algebraic group is the direct product of a connected semisimple Lie group and a connected abelian Lie group modulo some discrete central subgroup. Additionally, every connected semisimple Lie group coincides with its commutator subgroup. If it is even linear, then it has finite center [11] and, therefore [4, finite commutator length. Consequently, we are here precisely in the setting of Theorem 4.9 and Corollary 4.10 
- Since the smallest subgroup of $\mathbf{G}^{n}$ containing $\mathbf{G}_{V}$ has to contain every $\left[\mathbf{G}_{V}\right]^{\bullet q}$ with $q \in \mathbb{N}$, it has to contain $\left[\mathbf{G}_{V}\right]^{\bullet} q\left(n-\delta_{V}\right)$, which, however, is already a Lie subgroup containing $\mathbf{G}_{V}$. Hence, $\left[\mathbf{G}_{V}\right]^{\bullet q\left(n-\delta_{V}\right)}$ is precisely that smallest subgroup generated by $\mathbf{G}_{V}$. Corollary 4.10yields the assertion. Since $\operatorname{span}_{\mathbb{R}} V=\mathbb{R}^{n}$ implies that $V$ is rich, the condition for $\left[\mathbf{G}_{V}\right]^{\bullet q\left(n-\delta_{V}\right)}=\mathbf{G}^{n}$ is clear as well.

qed

Finally, we remark that every compact Lie group is a reductive linear algebraic group.

\section{$5 \quad$ Holonomical Independence}

The aim of the present section is to investigate in detail which parallel transports may be assigned by smooth connections to certain sets of paths. From now on, G is a (real or complex) Lie group.

Definition 5.1 Let $\gamma=\left(\gamma_{1}, \ldots, \gamma_{n}\right) \subseteq \mathcal{P}$ be some tuple of paths and let $\iota$ be some ultralocal trivialization. Then $\gamma$ is called

1. holonomically isolated iff

- the paths in $\gamma$ are non-selfintersecting,

- $(\gamma(0) \cup \gamma(1)) \cap \operatorname{int} \gamma=\varnothing$ and

- for every closed subset $K$ of $M$ with $K \cap \operatorname{int} \gamma=\varnothing$, for every $A \in \mathcal{A}$ and every $\left(g_{1}, \ldots, g_{n}\right) \in \mathcal{A}_{\boldsymbol{\gamma}}^{\iota} \subseteq \mathbf{G}^{n}$ there is an $A^{\prime} \in \mathcal{A}$ such that

- $h_{\gamma_{i}}^{\iota}\left(A^{\prime}\right)=g_{i}$ for $i=1, \ldots, n$ and

- $A^{\prime}$ and $A$ coincide on $K$;

2. holonomically independent iff for every $\left(g_{1}, \ldots, g_{n}\right) \in \mathbf{G}^{n}$ there is some $A \in \mathcal{A}$, such that $h_{\gamma_{i}}^{\iota}(A)=g_{i}$ for all $i=1, \ldots, n$;

3. strongly holonomically independent iff it is holonomically independent and holonomically isolated.

Note that int $\gamma$ is defined by $\gamma((0,1)):=\bigcup_{i} \gamma_{i}((0,1))$, which is generally not the interior of the image im $\gamma:=\bigcup_{i} \gamma_{i}([0,1])$ of $\gamma$. Analogously, $\gamma(0)$ and $\gamma(1)$ are here the sets formed by the components of the tuples $\left(\gamma_{1}(0), \ldots, \gamma_{n}(0)\right)$ and $\left(\gamma_{1}(1), \ldots, \gamma_{n}(1)\right)$, respectively.

We have obviously

Lemma 5.1 The notions of Definition 5.1 do not depend on the chosen trivialization $\iota$.

Before we come to the next statements, let us first discuss the relevance of the preceding definition comparing, for simplicity, the "normal" holonomy independence and the strong holonomy independence. In the case of the weaker, i.e. normal independence, it cannot a priori be excluded that products of independent sets of paths are not independent, even if they are non-overlapping outside their endpoints: Let $\vec{g}:=\left(g_{1}, \ldots, g_{n}\right) \in \mathbf{G}^{n}$ and let $\boldsymbol{\gamma}$ and $\boldsymbol{\delta}$ be given. Moreover, let $\vec{g}=\vec{g}^{\prime} \vec{g}^{\prime \prime}$. Now, it is, by independence, possible to find some $A^{\prime}$ with $h_{\boldsymbol{\gamma}}\left(A^{\prime}\right)=\vec{g}^{\prime}$ and some $A^{\prime \prime}$ with $h_{\boldsymbol{\delta}}\left(A^{\prime \prime}\right)=\vec{g}^{\prime \prime}$, but this does not directly imply the existence of some $A^{\prime \prime \prime}$ with $h_{\gamma \circ \delta}\left(A^{\prime \prime \prime}\right)=\vec{g}^{\prime} \vec{g}^{\prime \prime}$, maybe just by setting $\left.A^{\prime \prime \prime}\right|_{\operatorname{im} \gamma}=A^{\prime}$ and $\left.A^{\prime \prime \prime}\right|_{\operatorname{im} \delta}=A^{\prime \prime}$. To make this possible, i.e., to get a smooth $A^{\prime \prime \prime}$, one has to control at least the interface between $\gamma$ and $\boldsymbol{\delta}$ what precisely is done for the strong form of holonomy independence. Here we explicitly demand that the independence condition does not touch the values of the connections at the endpoints of the paths. Making this heuristic discussion more precise will be the goal of the next few claims. 
Lemma 5.2 Let $\gamma^{1}, \ldots, \gamma^{J}$ be finitely many holonomically isolated tuples of paths and $\operatorname{set}^{3} \gamma:=\bigcup_{j} \gamma^{j}$. Assume, moreover, im $\gamma^{j} \cap \operatorname{int} \gamma^{j^{\prime}}$ is empty unless $j=j^{\prime}$. Then we have:

- $\gamma$ is holonomically isolated with $\mathcal{A}_{\boldsymbol{\gamma}}^{\iota}=\times_{j} \mathcal{A}_{\boldsymbol{\gamma}^{j}}^{\iota}$ for every trivialization $\iota$.

- $\gamma$ is strongly holonomically independent, if each $\gamma^{j}$ is.

Note that if, additionally, all $\mathcal{A}_{\boldsymbol{\gamma}^{j}}^{\iota}$ are independent of the trivialization $\iota$, then $\mathcal{A}_{\boldsymbol{\gamma}}^{\iota}$ is so as well.

Proof • $\gamma$ is holonomically isolated.

The non-selfintersection property is obvious.

Next, we have $\left(\gamma^{j}(0) \cup \gamma^{j}(1)\right) \cap \operatorname{int} \gamma^{j^{\prime}} \subseteq \operatorname{im} \gamma^{j} \cap \operatorname{int} \gamma^{j^{\prime}}=\varnothing$ for $j \neq j^{\prime}$, hence

$$
\begin{aligned}
(\gamma(0) \cup \gamma(1)) \cap \operatorname{int} \gamma & =\bigcup_{j} \cup_{j^{\prime}}\left(\gamma^{j}(0) \cup \gamma^{j}(1)\right) \cap \operatorname{int} \gamma^{j^{\prime}} \\
& =\bigcup_{j}\left(\gamma^{j}(0) \cup \gamma^{j}(1)\right) \cap \operatorname{int} \gamma^{j} \\
& =\varnothing
\end{aligned}
$$

by the isolation property of every $\gamma^{j}$.

The third condition can be proven inductively. For this, let there be given some closed $K \subseteq M \backslash$ int $\boldsymbol{\gamma}$, some $A \in \mathcal{A}$ and some $g_{i}^{j} \in \mathbf{G}$ corresponding to the paths $\gamma_{i}^{j} \in \gamma$, such that $\vec{g} \in \times_{j} \mathcal{A}_{\gamma^{j}}$. (We fixed some trivialization $\iota$, but drop here the corresponding superscripts for $\mathcal{A}_{\ldots}$ and $h_{A}$.) We set $A_{0}:=A$ and choose some $A_{j} \in \mathcal{A}$ for $j=1, \ldots, J$ such that $h_{A_{j}}\left(\gamma_{i}^{j}\right)=g_{i}^{j}$ for all $i$ and that $A_{j}$ and $A_{j-1}$ coincide on $K \cup \bigcup_{j^{\prime} \neq j}$ im $\gamma^{j^{\prime}}$. The last union is compact and, by assumption, disjoint to int $\gamma^{j}$. Now let $A^{\prime}:=A_{J}$. Since, of course, $\mathcal{A}_{\boldsymbol{\gamma}} \subseteq \mathrm{X}_{j} \mathcal{A}_{\boldsymbol{\gamma}^{j}}$, we see that $A^{\prime}$ has the desired properties for $\gamma$ to be holonomically isolated. But, moreover, the construction showed also that $\mathrm{X}_{j} \mathcal{A}_{\boldsymbol{\gamma}^{j}} \subseteq \mathcal{A}_{\boldsymbol{\gamma}}$, hence their equality.

- The statement for the strong holonomy independence is now clear. qed

Lemma 5.3 Let $A \in \mathcal{A}$ be some smooth connection and $\gamma=\left(\gamma_{1}, \ldots, \gamma_{n}\right)$ be some tuple of non-selfintersecting paths. We assume that every $\gamma_{i}$ has an open neighbourhood $U_{i} \subseteq M$ that contains $\gamma_{i}$ as an embedding. Moreover, let all the $U_{i}$ be mutually disjoint.

Then $\gamma$ is strongly holonomically independent.

Proof Let $K$ be closed with $K \cap$ int $\gamma=\varnothing$. Thus, $M \backslash K$ is an open neighbourhood for int $\gamma$. Hence, every $U_{i}^{\prime}:=U_{i} \cap(M \backslash K)$ contains $\left.\gamma_{i}\right|_{(0,1)}$ as an embedding. Moreover, $U_{i}^{\prime}$ and $U_{j}^{\prime}$ are disjoint for $i \neq j$.

Let now $A \in \mathcal{A}$ and choose some ultralocal trivialization $\iota$. Then in the case of compact $\mathbf{G}$ it is well known that for every $\vec{g} \in \mathbf{G}^{n}$ there is some $A^{\prime} \in \mathcal{A}$ with $h_{A^{\prime}}^{\iota}(\gamma)=\vec{g}$, such that $A^{\prime}$ and $A$ coincide outside $\bigcup_{i} U_{i}^{\prime}$. The proof in the general case including non-compact $\mathbf{G}$ is not really more difficult. For completeness it is given in Appendix A Moreover, by $\bigcup_{i} U_{i}^{\prime}=\bigcup_{i}\left(U_{i} \cap(M \backslash K)\right) \subseteq M \backslash K$, the two connections $A$ and $A^{\prime}$ coincide at least on $K$, whence $\gamma$ is holonomically isolated. The holonomical independence is now obvious.

qed

Lemma 5.4 Let $\gamma$ be some finite tuple of non-selfintersecting paths in $M$, whereas the image of $\gamma$ is contained in some open set $U$, such that $P$ restricted to $\pi^{-1}(U)$

\footnotetext{
${ }^{3}$ Here, the "union" of tuples is given in the natural way: Simply list all components of all the $\gamma^{j}$. To be extremely precise: $\gamma=\left(\gamma_{1}^{1}, \ldots, \gamma_{N_{1}}^{1}, \ldots, \gamma_{1}^{J}, \ldots, \gamma_{N_{J}}^{J}\right)$ if $\gamma^{j}=\left(\gamma_{1}^{j}, \ldots, \gamma_{N_{j}}^{j}\right)$ for all $j$.
} 
is trivial. Additionally, assume $(\gamma(0) \cup \gamma(1)) \cap \operatorname{int} \gamma=\varnothing$. Moreover, let $I=\left[I_{-}, I_{+}\right]$be some closed interval in $[0,1]$ such that $\gamma\left(\left[0, I_{-}\right]\right), \gamma(\operatorname{int} I)$ and $\gamma\left(\left[I_{+}, 1\right]\right)$ are mutually disjoint. Assume finally that $\left.\gamma\right|_{I}$ is holonomically isolated and $\mathcal{A}_{\gamma}^{\iota} \subseteq \mathcal{A}_{\gamma \mid I}^{\iota}$ in some ultralocal trivialization $\iota$ that is smooth on $U$. Then we have:

1. $\mathcal{A}_{\gamma}^{\iota}=\mathcal{A}_{\left.\gamma\right|_{I}}^{\iota}$.

2. If $\mathcal{A}_{\left.\boldsymbol{\gamma}\right|_{I}}^{\iota}$ is a subgroup of $\mathbf{G}^{n}$, then $\boldsymbol{\gamma}$ is holonomically isolated.

Proof 1. $\mathcal{A}_{\gamma}^{\iota}=\mathcal{A}_{\left.\gamma\right|_{I}}^{\iota}$

Since im $\gamma$ is contained in $U$ and there is some trivialization $\iota$ that is smooth on $U$, there is a connection $A_{0} \in \mathcal{A}$ with trivial parallel transports w.r.t. $\iota$ along $\gamma$. Since $\left.\gamma\right|_{I}$ is holonomically isolated and since $\left.\gamma(\operatorname{int} I) \equiv \operatorname{int} \gamma\right|_{I}$ and the compact set $\gamma([0,1] \backslash \operatorname{int} I)$ are disjoint, we have for every $\vec{g} \in \mathcal{A}_{\gamma \mid{ }_{I}}^{\iota}$ some connection whose parallel transports on $\left.\gamma\right|_{I}$ equal $\vec{g}$ and on $\left.\gamma\right|_{\left[0, I_{-}\right]}$and $\left.\gamma\right|_{\left[I_{+}, 1\right]}$ equal those of $A_{0}$ being trivial. Consequently, $\mathcal{A}_{\left.\gamma\right|_{I}}^{\iota} \subseteq \mathcal{A}_{\gamma}^{\iota} \subseteq \mathcal{A}_{\left.\gamma\right|_{I}}^{\iota}$.

2. $\quad \gamma$ is holonomically isolated.

Before we start, we define $\gamma_{-}$by $\left.\gamma\right|_{\left[0, I_{-}\right]}$and $\gamma_{+}$by $\left.\gamma\right|_{\left[I_{+}, 1\right]}$.

- $h_{A}^{\iota}\left(\gamma_{-}\right) \in \mathcal{A}_{\left.\gamma_{I}\right|_{I}}^{\iota}$ for all $A \in \mathcal{A}$

Let $A$ be some smooth connection. Since, by assumption, the compact sets im $\gamma_{-}$and im $\gamma_{+}$are disjoint, there is a smooth function $f$ being 1 on im $\gamma_{+}$ with supp $f \subseteq U \backslash \operatorname{im} \gamma_{-}$. Define $A^{\prime}$ by $(1-f) A$ on $U$ (w.r.t. the fixed trivialization $\iota$ on $U$ ) and by $A$ outside. Of course, this is a connection with $h_{A^{\prime}}^{\iota}\left(\boldsymbol{\gamma}_{-}\right)=h_{A}^{\iota}\left(\boldsymbol{\gamma}_{-}\right)$and $h_{A^{\prime}}^{\iota}\left(\boldsymbol{\gamma}_{+}\right)=\left(e_{\mathbf{G}}, \ldots, e_{\mathbf{G}}\right)$. Consequently, $h_{A^{\prime}}^{\iota}(\gamma)=$ $h_{A^{\prime}}^{\iota}\left(\gamma_{-}\right) h_{A^{\prime}}^{\iota}\left(\left.\gamma\right|_{I}\right)$, hence $h_{A}^{\iota}\left(\gamma_{-}\right)=h_{A^{\prime}}^{\iota}\left(\gamma_{-}\right)=h_{A^{\prime}}^{\iota}(\gamma) h_{A^{\prime}}^{\iota}\left(\left.\gamma\right|_{I}\right)^{-1} \in \mathcal{A}_{\left.\gamma\right|_{I}}^{\iota}$ by the group property of $\mathcal{A}_{\left.\boldsymbol{\gamma}\right|_{I}}^{\iota}$ and $\mathcal{A}_{\boldsymbol{\gamma}}^{\iota}=\mathcal{A}_{\gamma_{\left.\right|_{I}}}^{\iota}$.

- $h_{A}^{\iota}\left(\gamma_{+}\right) \in \mathcal{A}_{\left.\gamma\right|_{I}}^{\iota}$ for all $A \in \mathcal{A}$

This is shown completely analogously to $h_{A}^{\iota}\left(\gamma_{-}\right) \in \mathcal{A}_{\gamma_{I}}^{\iota}$.

- $h_{A}^{\iota}\left(\gamma_{-}\right)^{-1} \mathcal{A}_{\gamma}^{\iota} h_{A}^{\iota}\left(\gamma_{+}\right)^{-1} \subseteq \mathcal{A}_{\gamma_{\left.\right|_{I}}}^{\iota}$ for all $A \in \mathcal{A}$

Using $\mathcal{A}_{\boldsymbol{\gamma}}^{\iota}=\mathcal{A}_{\left.\boldsymbol{\gamma}\right|_{I}}^{\iota}$ and the group property of $\mathcal{A}_{\left.\boldsymbol{\gamma}\right|_{I}}^{\iota}$ again, we get the desired relation.

- Isolation property of $\gamma$

Let $K \subseteq M$ be closed with $K \cap \operatorname{int} \gamma=\varnothing$. Define $K^{\prime}:=K \cup \operatorname{im} \gamma_{-} \cup \operatorname{im} \gamma_{+}$. Obviously, $K^{\prime}$ is closed. Let now $x \in K^{\prime} \cap$ int $\left.\gamma\right|_{I}$. Since, by assumption, $K \cap$ int $\gamma=\varnothing$, we have $x \in \gamma([0,1] \backslash$ int $I)$. This is a contradiction to $\gamma(\operatorname{int} I) \cap \gamma([0,1] \backslash$ int $I)=\varnothing$. Hence, $K^{\prime} \cap$ int $\left.\gamma\right|_{I}=\varnothing$.

Let now $A \in \mathcal{A}$ and $\vec{g}:=\left(g_{1}, \ldots, g_{n}\right) \in \mathcal{A}_{\boldsymbol{\gamma}}^{\iota}$. Then, since - as shown above $h_{A}^{\iota}\left(\gamma_{-}\right)^{-1} \mathcal{A}_{\boldsymbol{\gamma}}^{\iota} h_{A}^{\iota}\left(\gamma_{+}\right)^{-1} \subseteq \mathcal{A}_{\left.\boldsymbol{\gamma}\right|_{I}}^{\iota}$ and since $\left.\gamma\right|_{I}$ is assumed holonomically isolated, there is some $A^{\prime \prime} \in \mathcal{A}$, such that

- $h_{A^{\prime \prime}}^{\iota}\left(\left.\gamma\right|_{I}\right)=h_{A}^{\iota}\left(\left.\gamma\right|_{\left[0, I_{-}\right]}\right)^{-1} \vec{g} h_{A}^{\iota}\left(\left.\gamma\right|_{\left[I_{+}, 1\right]}\right)^{-1}$ and

- $A^{\prime \prime}$ and $A$ coincide on $K^{\prime}$.

Consequently, $h_{\gamma}^{\iota}\left(A^{\prime \prime}\right)=\vec{g}$ as well as $A^{\prime \prime}$ and $A$ coincide on $K \subseteq K^{\prime}$.

qed

\section{Regularity and Consistent Parametrization}

In this section we get closer to the case of webs. First we recall (and slightly extend) the notions of consistent parametrization and regularity [3]. The latter one means, in particular, that, given a set of paths, around regular points there are no intersections between different paths, unless they are locally identical. The former one means that intersections of paths are 


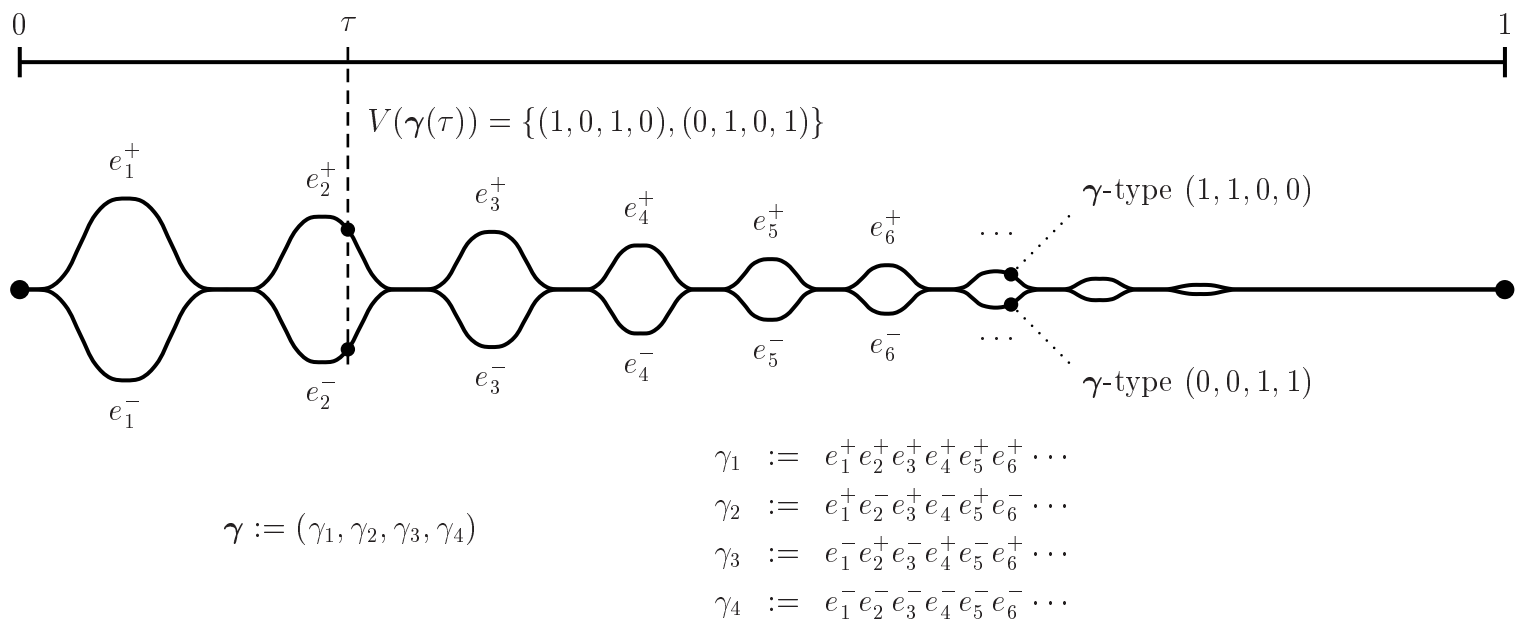

Figure 1: A special set of paths owing to Baez and Sawin [2]

allowed only at those points where the paths under consideration have identical parameter values. Afterwards we introduce the type of points w.r.t. certain paths in generalization of the similar notion settled by Baez and Sawin 3 .

Definition 6.1 An $n$-tuple $\gamma=\left(\gamma_{1}, \ldots, \gamma_{n}\right)$ of paths is called consistently parametrized iff we have for all $i, j=1, \ldots, n$

$$
\gamma_{i}\left(t^{\prime}\right)=\gamma_{j}\left(t^{\prime \prime}\right) \Longrightarrow t^{\prime}=t^{\prime \prime}
$$

Definition 6.2 Let $\gamma$ be some $n$-tuple of paths.

- A point $x \in M$ is called $\gamma$-regular iff $x$ is not the image of an endpoint or nondifferentiable point of $\gamma$ and there is a neighbourhood of $x$ whose intersection with im $\gamma$ is an embedded interval.

- $\tau \in[0,1]$ is called $\boldsymbol{\gamma}$-regular iff $\gamma(\tau)$ is $\boldsymbol{\gamma}$-regular for all $\gamma \in \boldsymbol{\gamma}$.

We have immediately

Lemma 6.1 For every consistently parametrized $n$-tuple $\gamma$ of paths in $M$, the set of $\gamma$ regular parameter values is open in $[0,1]$.

Definition 6.3 Let $\gamma=\left(\gamma_{1}, \ldots, \gamma_{n}\right)$ be some $n$-tuple of paths.

- For every $x \in M$ we define the $\boldsymbol{\gamma}$-type $v(x) \in \mathcal{V}_{n}$ of $x$ by

$$
v(x)_{i}:=\left\{\begin{array}{ll}
1 & \text { if } x \in \operatorname{im} \gamma_{i} \\
0 & \text { if } x \notin \operatorname{im} \gamma_{i}
\end{array} .\right.
$$

- For every consistently parametrized $\gamma$ we define

$$
V_{\gamma}:=\bigcup_{\tau \in[0,1], \tau} \gamma \text {-regular } V(\gamma(\tau)) \text {. }
$$

For consistently parametrized $\gamma$, obviously, $V(\gamma(\tau))$ is the set of all $\gamma$-types in $\gamma(\tau)$. Note, moreover, that in general the set $V_{\gamma}$ of types in $\gamma$ and the splitting $V(\gamma)$ for $\gamma$ do not coincide. For instance, we have in the case of Figure 1]

$$
\begin{aligned}
V_{\gamma} & =\{(1,1,0,0),(1,0,1,0),(0,1,0,1),(0,0,1,1)\} \\
V(\gamma) & =\{(1,0,0,0),(0,1,0,0),(0,0,1,0),(0,0,0,1)\} .
\end{aligned}
$$


In a certain sense, $V_{\gamma}$ is finer. $V(\gamma)$ only looks whether two whole paths are equal or not. $V_{\gamma}$ looks closer at the image points of $\gamma$.

Let us now study first consequences of the just introduced notions for parallel transports.

Lemma 6.2 Let $\gamma$ be some consistently parametrized tuple of paths in $M$. Assume that im $\gamma$ is contained in some open $U$, such that $P(M, \mathbf{G})$ is trivial when restricted to the preimage of $U$. Additionally, let $I \subseteq[0,1]$ be some interval whose endpoints are $\gamma$-regular. Then we have $\mathcal{A}_{\left.\gamma\right|_{I}}^{\iota} \subseteq \mathcal{A}_{\gamma}^{\iota}$ for all ultralocal trivializations $\iota$ that are smooth on $U$.

Proof Let $\vec{g} \in \mathcal{A}_{\left.\gamma\right|_{I}}^{\iota}$, i.e. there is some smooth $A \in \mathcal{A}$, such that $h_{A}^{\iota}\left(\left.\gamma\right|_{I}\right)=\vec{g}$. Next, let $I=\left[I_{-}, I_{+}\right]$. Since the set of $\gamma$-regular parameter values is open in $(0,1)$, there is some $\varepsilon>0$ such that $\left[I_{-}-2 \varepsilon, I_{-}\right]$and $\left[I_{+}, I_{+}+2 \varepsilon\right]$ are $\gamma$-regular intervals. Set $J_{-\lambda}:=\left[I_{-}-(\lambda+1) \varepsilon, I_{-}-\lambda \varepsilon\right]$ and $J_{+\lambda}:=\left[I_{+}+\lambda \varepsilon, I_{+}+(\lambda+1) \varepsilon\right]$ for $\lambda \geq 0$. Finally, we set $J_{-\infty}:=\left[0, I_{-}-2 \varepsilon\right]$ and $J_{+\infty}:=\left[I_{+}+2 \varepsilon, 1\right]$.

Because $\gamma$ is consistently parametrized and $\gamma$ is continuous, the sets $\gamma\left(J_{-\infty}\right), \gamma(I)$ and $\gamma\left(J_{+\infty}\right)$ are disjoint and compact. Consequently, there is some smooth function $f$ on $M$ that has support on $U \backslash \gamma(I)$ and equals 1 on $\gamma\left(J_{-\infty}\right) \cup \gamma\left(J_{+\infty}\right)$. We now define some connection $A^{\prime}$ by $(1-f) A$ on $U$ and by $A$ outside of $U$. Obviously, $A^{\prime} \in \mathcal{A}$.

Observe now, that, by regularity, two paths in $\gamma$ coincide on $J_{ \pm 0}$ iff they coincide on $J_{ \pm 1}$. Moreover, they coincide iff their images have just a common point. Set $\boldsymbol{\delta}=\left\{\delta_{1}, \ldots, \delta_{l}\right\}:=\left.\left.\gamma\right|_{\text {int } J_{-1}} \cup \gamma\right|_{\text {int } J_{+1}}$, considered as sets. Choose now some disjoint open sets $V_{j}$ in $U$ that are disjoint to $\gamma\left([0,1] \backslash\left(\right.\right.$ int $J_{-1} \cup$ int $\left.\left.J_{+1}\right)\right)$, such that there are certain closed intervals $I_{j}$ in $J_{-1}$ or $J_{+1}$, respectively, such that $V_{j}$ is a neighbourhood for $\delta_{j}\left(I_{j}\right)$ and that im $\left.\delta_{j}\right|_{I_{j}} \cap V_{j}$ is embedded into $V_{j}$. Then, by Proposition A.1 there is some smooth connection $A^{\prime \prime} \in \mathcal{A}$, such that

- $h_{A^{\prime \prime}}^{\iota}\left(\left.\gamma\right|_{J_{-1}}\right)=h_{A^{\prime}}^{\iota}\left(\left.\gamma\right|_{J_{-0}}\right)^{-1}$;

- $h_{A^{\prime \prime}}^{\iota}\left(\left.\gamma\right|_{J_{+1}}\right)=h_{A^{\prime}}^{\iota}\left(\left.\gamma\right|_{J_{+0}}\right)^{-1}$;

- $A^{\prime}$ and $A^{\prime \prime}$ coincide outside $\bigcup V_{j}$.

Altogether, we have

$$
\begin{aligned}
& h_{A^{\prime \prime}}^{\iota}(\gamma)=h_{A^{\prime \prime}}^{\iota}\left(\left.\gamma\right|_{J_{-\infty}}\right) h_{A^{\prime \prime}}^{\iota}\left(\left.\gamma\right|_{J_{-1}}\right) h_{A^{\prime \prime}}^{\iota}\left(\left.\gamma\right|_{J_{-0}}\right) h_{A^{\prime \prime}}^{\iota}\left(\left.\gamma\right|_{I}\right) \text {. } \\
& \cdot h_{A^{\prime \prime}}^{\iota}\left(\left.\gamma\right|_{J_{+0}}\right) h_{A^{\prime \prime}}^{\iota}\left(\left.\gamma\right|_{J_{+1}}\right) h_{A^{\prime \prime}}^{\iota}\left(\left.\gamma\right|_{J_{+\infty}}\right) \\
& =h_{A^{\prime}}^{\iota}\left(\left.\gamma\right|_{J_{-\infty}}\right) h_{A^{\prime}}^{\iota}\left(\left.\gamma\right|_{J_{-0}}\right)^{-1} h_{A^{\prime}}^{\iota}\left(\left.\gamma\right|_{J_{-0}}\right) h_{A^{\prime}}^{\iota}\left(\left.\gamma\right|_{I}\right) \text {. } \\
& \cdot h_{A^{\prime}}^{\iota}\left(\left.\gamma\right|_{J_{+0}}\right) h_{A^{\prime}}^{\iota}\left(\left.\gamma\right|_{J_{+0}}\right)^{-1} h_{A^{\prime}}^{\iota}\left(\left.\gamma\right|_{J_{+\infty}}\right) \\
& =h_{A}^{\iota}\left(\left.\gamma\right|_{I}\right) \\
& =\vec{g} \text {. }
\end{aligned}
$$

Consequently, $\vec{g} \in \mathcal{A}_{\boldsymbol{\gamma}}^{\iota}$.

qed

Proposition 6.3 Let $\gamma:=\left(\gamma_{1}, \ldots, \gamma_{n}\right)$ be some consistently parametrized tuple of paths in $M$. Assume that im $\gamma$ is contained in some open $U$, such that $P(M, \mathbf{G})$ is trivial if restricted to the preimage of $U$. Moreover, let $\left(\tau_{k}\right)$ be some finite, strictly increasing sequence of $\boldsymbol{\gamma}$-regular parameter values in $[0,1]$. Then we have, if $\iota$ is any ultralocal trivialization being smooth on $U$ :

1. If $\mathcal{A}_{\boldsymbol{\gamma}}^{\iota}$ is a subgroup of $\mathbf{G}^{n}$ and equals $\prod_{k} \mathbf{G}_{V\left(\gamma\left(\tau_{k}\right)\right)}$, then $\boldsymbol{\gamma}$ is holonomically isolated.

2. $\mathcal{A}_{\boldsymbol{\gamma}}^{\iota}$ contains $\prod_{k} \mathbf{G}_{V\left(\gamma\left(\tau_{k}\right)\right)}$. 
Proof Let us fix for all $k$ some $\tau_{k}^{-}$and $\tau_{k}^{+}$with $0<\tau_{k}^{-}<\tau_{k}<\tau_{k}^{+}<1$, such that every $\tau \in\left[\tau_{k}^{-}, \tau_{k}^{+}\right]=: I_{k}$ is $\gamma$-regular. ${ }^{4}$ Moreover we demand $\tau_{k-1}^{+}<\tau_{k}^{-}$for all $k \neq 1$. Such $\tau_{k}^{ \pm}$always exist by the regularity of $\tau_{k}$. Again by regularity we have $\gamma_{i}(\tau)=\gamma_{j}(\tau)$ with $\tau \in I_{k}$ iff $\gamma_{i}\left(\tau_{k}\right)=\gamma_{j}\left(\tau_{k}\right)$, i.e., the restrictions of two paths to $I_{k}$ are identical iff their images are non-empty. This implies $V(\gamma(\tau))=V\left(\gamma\left(\tau_{k}\right)\right)$ (possibly up to the ordering) for all $\tau \in I_{k}$, hence $\mathbf{G}_{V(\gamma(\tau))}=\mathbf{G}_{V\left(\gamma\left(\tau_{k}\right)\right)}$ by Lemma 3.3. Additionally, we define $I:=\bigcup_{k} I_{k}$ and $J:=\bigcup_{k \neq 1}\left[t_{k-1}^{+}, t_{k}^{-}\right]$, i.e., $J$ contains all (closures of) intervals between the $I_{k} \mathrm{~s}$. Moreover, set $J_{0}:=\left[0, t_{1}^{-}\right]$and $J_{1}:=\left[t_{k_{\max }}^{+}, 1\right]$. Finally, we define the interval $L:=I \cup J=\left[t_{1}^{-}, t_{k_{\max }}^{+}\right]$.

Let now $\boldsymbol{\delta}^{k}$ be the set of all $\left.\gamma_{i}\right|_{I_{k}}, i=1, \ldots, n$, and $\boldsymbol{\delta}:=\bigcup_{k} \boldsymbol{\delta}^{k}$. Then im $\delta$ is compact for every $\delta \in \boldsymbol{\delta}$, and (im $\left.\delta^{\prime}\right) \cap\left(\operatorname{im} \delta^{\prime \prime}\right)$ is non-empty iff $\delta^{\prime}=\delta^{\prime \prime}$ for $\delta^{\prime}, \delta^{\prime \prime} \in \boldsymbol{\delta}$. Since every $\delta \in \delta$ is strongly holonomically independent by Proposition A.1 their union $\boldsymbol{\delta}$ is so by Lemma 5.2

Now we are prepared for the proofs of the assertions in the proposition:

1. Assume $\prod_{k} \mathbf{G}_{V\left(\boldsymbol{\gamma}\left(\tau_{k}\right)\right)}=\mathcal{A}_{\boldsymbol{\gamma}}^{\iota}$ being a subgroup of $\mathbf{G}^{n}$.

By consistent parametrization, the first two conditions for $\left.\gamma\right|_{L}$ to be holonomically isolated are fulfilled. To prove the third one, let $K$ be some closed subset of $M$ with $K \cap$ int $\left.\gamma\right|_{L}=\varnothing$ and let $A \in \mathcal{A}$ be arbitrary. Since $\gamma(J) \subseteq$ int $\left.\gamma\right|_{L}$ is compact, there is some smooth function $f$ on $M$ being 1 on $\gamma(J)$ with supp $f \subseteq U \cap(M \backslash K)$, hence $f=0$ on $K$. We define $A_{f}$ to be the connection that coincides on $U$ with $(1-f) A$ and equals $A$ outside.

Since $I_{k}$ is always $\boldsymbol{\gamma}$-regular, Lemma 6.2 yields $\mathcal{A}_{\left.\boldsymbol{\gamma}\right|_{L}} \subseteq \mathcal{A}_{\boldsymbol{\gamma}}^{\iota}=\prod_{k} \mathbf{G}_{V\left(\boldsymbol{\gamma}\left(\tau_{k}\right)\right)}$. Then for every $\left(g_{1}, \ldots, g_{n}\right) \in \prod_{k} \mathbf{G}_{V\left(\gamma\left(\tau_{k}\right)\right)} \supseteq \mathcal{A}_{\left.\gamma\right|_{L}}^{\iota}$ we have certain $\left(g_{1, k}, \ldots, g_{n, k}\right)$ in $\mathbf{G}_{V\left(\gamma\left(\tau_{k}\right)\right)}$ with $\left(g_{1}, \ldots, g_{n}\right)=\prod_{k}\left(g_{1, k}, \ldots, g_{n, k}\right)$. Since $\boldsymbol{\delta}$ is strongly holonomically independent and int $\boldsymbol{\delta} \subseteq \bigcup_{k}$ int $\left.\gamma\right|_{I_{k}}$, there is some $A^{\prime} \in \mathcal{A}$ such that, in particular,

- $h_{\delta}^{\iota}\left(A^{\prime}\right)=g_{i, k}$ for all $i, k$ and $\delta \in \boldsymbol{\delta}$ with $\delta=\left.\gamma_{i}\right|_{I_{k}}$;

- $A^{\prime}$ and $A_{f}$ coincide on $K \cup \gamma(J)$.

$h_{\delta}^{\iota}\left(A^{\prime}\right)$ is indeed well defined, because $\left.\gamma_{i^{\prime}}\right|_{I_{k^{\prime}}}=\delta=\left.\gamma_{i}\right|_{I_{k}}$ implies $k^{\prime}=k$, hence $\gamma_{i^{\prime}}\left(\tau_{k}\right)=\gamma_{i}\left(\tau_{k}\right)$ and thus $g_{i^{\prime}, k^{\prime}} \equiv g_{i^{\prime}, k}=g_{i, k}$ by the definition of $\mathbf{G}_{V\left(\gamma\left(\tau_{k}\right)\right)}$.

Since $A_{f}$ is zero on $\gamma(J)$, the parallel transports along all subpaths of $\gamma_{i}$ for the parameter intervals $\left[\tau_{k-1}^{+}, \tau_{k}^{-}\right]$with $k \neq 1$ are $e_{\mathbf{G}}$. Hence, by construction,

$$
h_{\left.\gamma_{i}\right|_{L}}^{\iota}\left(A^{\prime}\right)=\prod_{k} h_{\left.\gamma_{i}\right|_{I_{k}}}^{\iota}\left(A^{\prime}\right)=\prod_{k} g_{i, k}=g_{i}
$$

for all $i$. Consequently, we see first that $\mathcal{A}_{\left.\boldsymbol{\gamma}\right|_{L}}^{\iota}=\prod_{k} \mathbf{G}_{V\left(\boldsymbol{\gamma}\left(\tau_{k}\right)\right)}=\mathcal{A}_{\boldsymbol{\gamma}}^{\iota}$, and second that $\left.\gamma\right|_{L}$ is holonomically isolated.

Hence, $\gamma$ is holonomically isolated by Lemma 5.4.

2. Now we show $\prod_{k} \mathbf{G}_{V\left(\boldsymbol{\gamma}\left(\tau_{k}\right)\right)} \subseteq \mathcal{A}_{\boldsymbol{\gamma}}^{\iota}$.

In contrast to the step above, we now choose some smooth function $f$ on $M$ being 1 on the full im $\gamma$ with supp $f \subseteq U$. Analogously defining $A_{f}$ and choosing $A^{\prime}$ we get for every $\left(g_{1}, \ldots, g_{n}\right) \in \prod_{k} \mathbf{G}_{V\left(\gamma\left(\tau_{k}\right)\right)}$ some $A^{\prime} \in \mathcal{A}$ with $h_{\gamma_{i}}\left(A^{\prime}\right)=g_{i}$, because now $A^{\prime}$ is trivial on $\gamma\left(J_{0} \cup J \cup J_{1}\right)$.

qed

\section{Parallel Transports Along Webs}

We now recall the definition of tassels and webs owing to Baez and Sawin [3, 2].

\footnotetext{
${ }^{4}$ Remember that 0 and 1 are not $\gamma$-regular.
} 
Definition 7.1 - A finite ordered set $T=\left\{c_{1}, \ldots, c_{n}\right\}$ of paths is called tassel based on $p \in \operatorname{im} T$ iff the following conditions are met:

1. im $T$ lies in a contractible open subset of $M$.

2. $T$ can be consistently parametrized in such a way that $c_{i}(0)=p$ is the left endpoint of every path $c_{i}$.

3. Two paths in $T$ that intersect at a point other than $p$ intersect at a point other than $p$ in every neighborhood of $p$.

4. For every neighbourhood $U$ of $p$, any $T$-type which occurs at some regular point in im $T$ occurs at some regular point in $U \cap \operatorname{im} T$.

5. No two paths in $T$ have the same image.

- A finite collection $w=w^{1} \cup \cdots \cup w^{k}$ of tassels is called web iff for all $i \neq j$ the following conditions are met:

1. Any path in the tassel $w^{i}$ intersects any path in $w^{j}$, if at all, only at their endpoints.

2. There is a neighborhood of each such intersection point whose intersection with $\operatorname{im}\left(w^{i} \cup w^{j}\right)$ is an embedded interval.

3. $\quad$ im $w^{i}$ does not contain the base of $w^{j}$.

Next, we list some important properties of webs that can be derived immediately from statements in 3 .

Proposition 7.1 For every web $w$ the set $[0,1]_{\text {reg }}$ of $w$-regular parameter values is open and dense in $[0,1]$. Moreover, the function $V(w(\cdot)):[0,1]_{\text {reg }} \longrightarrow \mathcal{V}_{\# w}$, assigning to every $w$-regular $\tau$ its splitting, is locally constant.

Proof A slight modification of the proof of Lemma 1 in [3] yields that for every $c_{j} \in w$ the set of all $\tau$ in $[0,1]$ with $w$-regular $c_{j}(\tau)$ is open and dense in $[0,1]$. Since the intersection of finitely many open and dense subsets is again open and dense ${ }^{5}$, $[0,1]_{\text {reg }}$ is open and dense in $[0,1]$. The second assertion is obvious.

qed

Lemma 7.2 For every web $w$ the set $V_{w}$ of $w$-types occurring in $w$ is rich.

Proof Since $w=\left\{c_{1}, \ldots, c_{n}\right\}$ is a web, for $i \neq j$ there is some $\tau \in[0,1]$ with $c_{i}(\tau) \neq c_{j}(\tau)$. By Proposition 7.1 there is even a regular $\tau$ with this property, thus $v\left(c_{i}(\tau)\right) \in V_{w}$. By the consistent parametrization we have $v\left(c_{i}(\tau)\right)_{i}=1 \neq 0=v\left(c_{i}(\tau)\right)_{j}$, i.e., the first richness condition is fulfilled. The second is trivial.

qed

For the following lemma, we still have to define the set

$$
\mathcal{V}(w):=\bigcap_{\tau \in(0,1]} \bigcup_{\sigma \in[0, \tau]_{\mathrm{reg}}}\{V(w(\sigma))\}
$$

of all those ("regular") splittings $V(w(\sigma))$ that appear in every neighbourhood of 0 . Here, $I_{\text {reg }}$ denotes the set of $w$-regular elements in an arbitrary interval $I \subseteq[0,1]$.

Lemma 7.3 Let $w$ be a web. Then for all $v \in V_{w}$ there is some $V \in \mathcal{V}(w)$ with $v \in V$. In particular, $\mathcal{V}(w)$ is nonempty (if $w$ is nonempty).

\footnotetext{
${ }^{5}$ Let $X_{1}, X_{2}$ be open and dense subsets in some topological space $X$. Then, of course, $X_{1} \cap X_{2}$ is open again. Assume $X_{1} \cap X_{2}$ were not dense in $X$. Then there is some $x \in X$ and some open neighbourhood $U$ of $x$ in $X$, such that $U \cap\left(X_{1} \cap X_{2}\right)=\varnothing$. Since $X_{1}$ is dense, there is some $x_{1} \in U \cap X_{1}$. Since $X_{2}$ is dense, the open neighbourhood $U \cap X_{1}$ of $x_{1}$ must contain some $x_{2} \in X_{2}$. Consequently, $x_{2} \in U \cap X_{1} \cap X_{2}=\varnothing$. Contradiction. The case of finitely many $X_{i}$ is now clear.
} 
Proof For every $v \in V_{w}$, by definition of a web, there is a sequence $\tau_{i} \rightarrow 0$ in $[0,1]_{\text {reg }}$ with $v \in V\left(w\left(\tau_{i}\right)\right) \subseteq \mathcal{V}_{\# w}$ for all $i$. Since $\mathcal{V}_{\# w}$ is finite, there is some $V \subseteq \mathcal{V}_{\# w}$ and some infinite subsequence $\tau_{i^{\prime}} \rightarrow 0$ with $V=V\left(w\left(\tau_{i^{\prime}}\right)\right) \ni v$ for all $i^{\prime}$. By definition, $V \in \mathcal{V}(w)$.

qed

Corollary 7.4 $\bigcup_{V \in \mathcal{V}(w)} V$ equals $V_{w}$ for every web $w$ and is rich.

Let us now state the main result of our article.

Theorem 7.5 Let $\mathbf{G}$ be a connected reductive (real or complex) linear algebraic group and let $T$ be some tassel. Then for every $t \in(0,1]$ there is some $t^{\prime} \in(0, t]$, such that for every $0 \leq \tau \leq t^{\prime}$ and every ultralocal trivialization $\iota$

- $\left.T\right|_{[\tau, t]}$ is holonomically isolated;

- $\mathcal{A}_{\left.T\right|_{[\tau, t]}}^{\iota}$ is a Lie subgroup of $\mathbf{G}^{n}$ and equals $\left[\mathbf{G}_{V}\right]^{\bullet q(\# T)}$.

Recall that every linear algebraic group is a Lie group and that, in particular, every compact Lie group is a reductive linear algebraic group. Hence, the assertion of the theorem above holds for all connected compact Lie groups $\mathbf{G}$.

A part of the following proof is owing to $[3]$.

Proof - Choice of $t^{\prime}$

Denote the paths in $T$ by $c_{1}, \ldots, c_{n}$ and fix some ultralocal trivialization $\iota$ being smooth on some open neighbourhood of im $T$. Fix, additionally, some ordering of $V:=V_{T}$. Since for every $v \in V$ there is some $V^{\prime} \in \mathcal{V}(T)$ with $v \in V^{\prime}$, there is some finite sequence $V^{(s)}$ in $\mathcal{V}(T)$ with $\prod_{s=1}^{S} \mathbf{G}_{V^{(s)}} \supseteq \mathbf{G}_{V}$. Let now $\left(V^{(s)}\right)_{s=1}^{S q(n)} \subseteq \mathcal{V}_{n}$ be the $q(n)$-times repeated sequence of these elements in $\mathcal{V}(T)$ and set $t_{S q(n)+1}:=t$. Starting with $s=S q(n)$, we choose inductively some $T$ regular $t_{s} \in\left(0, t_{s+1}\right)$, such that $V\left(T\left(t_{s}\right)\right)=V^{(s)}$. By definition of $\mathcal{V}(T)$, such a $t_{s}$ always exist. Finally, we choose some regular $t^{\prime}<t_{1}$.

- $\prod_{s=1}^{S q(n)} \mathbf{G}_{V^{(s)}}=\left[\mathbf{G}_{V}\right]^{\bullet q(n)}$

Let $v \in V^{(s)} \in \mathcal{V}(T)$ for some $s$. Then $v \in V$, i.e. $\mathbf{G}_{v} \subseteq\left[\mathbf{G}_{V}\right]^{\bullet q(n)}$. By the group property of the right-hand side, we have $\prod_{s=1}^{S q(n)} \mathbf{G}_{V^{(s)}} \subseteq\left[\mathbf{G}_{V}\right]^{\bullet q(n)}$. The opposite relation comes from $\prod_{s=1}^{S} \mathbf{G}_{V^{(s)}} \supseteq \mathbf{G}_{V}$ together with the definition of $\left(V^{(s)}\right)$ as a $q(n)$-fold repetition of the first $S$ sets.

- $\mathcal{A}_{\left.T\right|_{[\tau, t]}}^{\iota} \supseteq\left[\mathbf{G}_{V}\right]^{\bullet q(n)}$ for $\tau \leq t^{\prime}$

By the very definition of a tassel, $\left.T\right|_{[\tau, t]}$ fulfills the requirements of Proposition 6.3. Moreover, $\left(t_{s}\right)_{s=1}^{S q(n)}$ is a strictly increasing sequence of $T$-regular values in $[\tau, t]$. Hence, by the construction of this sequence and by the previous item, we have $\mathcal{A}_{\left.T\right|_{[\tau, t]}} \supseteq\left[\mathbf{G}_{V}\right]^{\bullet q(n)}$.

- $\left[\mathbf{G}_{V}\right]^{\bullet q(n)}$ is a Lie subgroup of $\mathbf{G}^{n}$

According to Lemma [7.2, the set of types in every tassel is rich. Proposition 4.11 guarantees now that $\left[\mathbf{G}_{V}\right]^{\bullet q(n)}$ is even a Lie subgroup of $\mathbf{G}^{n}$.

- $\mathcal{A}_{\left.T\right|_{I}}^{\iota} \subseteq\left[\mathbf{G}_{V}\right]^{\bullet q(n)}$ for every nontrivial interval $I \subseteq[0, t]$, where $\left.T\right|_{I}$ consists of smooth immersive paths only

We have to prove that $h_{A}^{\iota}\left(\left.T\right|_{I}\right) \in\left[\mathbf{G}_{V}\right]^{\bullet q(n)}$ for all $A \in \mathcal{A}$. For this, consider the map

$$
\begin{array}{ccc}
H: I \times I & \longrightarrow & \mathbf{G}^{n} . \\
\left(\tau_{1}, \tau_{2}\right) & \longmapsto & h_{A}^{\iota}\left(\left.T\right|_{\left[\min I, \tau_{1}\right]}\right)^{-1} h_{A}^{\iota}\left(\left.T\right|_{\left[\min I, \tau_{2}\right]}\right)
\end{array}
$$


Since $A$ and $\iota$ are smooth on $U$ containing im $T$, the map $H$ is smooth. Moreover, if $\left(\tau_{1}, \tau_{2}\right)$ (or $\left(\tau_{2}, \tau_{1}\right)$ ) is some interval of $T$-regular parameters only (which implies that every element in it has the same type, say, $v)$, then $H\left(\tau_{1}, \tau_{2}\right) \in \mathbf{G}_{v}$, hence $\dot{H}\left(\tau_{1}, \tau_{2}\right) \in \mathcal{L} \mathbf{G}_{v}$ where the dot means differentiation w.r.t. the $\tau_{2}$-coordinate. On the other hand, $\dot{H}\left(\tau_{1}, \tau_{1}\right)$ equals (up to the $\left.\operatorname{sign}\right)\left.A\right|_{T\left(\tau_{1}\right)}(\dot{T})$, where $\dot{T}$ is the $n$-tuple of tangential vectors on $T$ and $\left.A\right|_{T\left(\tau_{1}\right)}$ is given naturally.

Since we know that $\left[\mathbf{G}_{V}\right]^{\bullet q(n)}$ is a Lie group and since, as one sees immediately, $\mathbf{G}_{v}$ is Lie subgroup of $\left[\mathbf{G}_{V}\right]^{\bullet q(n)}$, the Lie algebra $\mathcal{L} \mathbf{G}_{v}$ is a Lie subalgebra of $\mathcal{L}\left(\left[\mathbf{G}_{V}\right]^{\bullet q(n)}\right)$. Consequently, $\left.A\right|_{T(\cdot)}(\dot{T})$ can be regarded as a smooth function from $I$ to $\mathcal{L} \mathbf{G}^{n}$ with values in $\mathcal{L}\left(\left[\mathbf{G}_{V}\right]^{\bullet q(n)}\right)$ - at least for those parameter values that are $T$-regular. However, since the set of $T$-regular points is open and dense in $I$ and $A$ is smooth, $\left.A\right|_{T(\cdot)}(\dot{T})$ values in $\mathcal{L}\left(\left[\mathbf{G}_{V}\right]^{\bullet q(n)}\right)$ everywhere. Since $h_{A}^{\iota}\left(\left.T\right|_{I}\right)$ is simply the path-ordered exponential of $\left.A\right|_{T(\cdot)}(\dot{T})$ integrated along $I$, it is contained in $\left[\mathbf{G}_{V}\right]^{\bullet q(n)}$.

- $\mathcal{A}_{\left.T\right|_{[\tau, t]} ^{\iota}}^{\iota} \subseteq\left[\mathbf{G}_{V}\right]^{\bullet q(n)}$ for $\tau \leq t^{\prime}$

Since we consider piecewise smooth and immersive paths from the very beginning, there are at most finitely many non-differentiability points in any finite set of paths. Hence, we can decompose $[\tau, t]$ into a finite set $I_{k}$ of intervals, such that $\left.T\right|_{[\tau, t]}=\left.\prod T\right|_{I_{k}}$, where each $\left.T\right|_{I_{k}}$ consists of smooth and immersive paths only. Since $h_{A}^{\iota}\left(\left.T\right|_{[\tau, t]}\right)$ is now the product of these $h_{A}^{\iota}\left(\left.T\right|_{I_{k}}\right)$, we get the assertion by the previous step and the group property of $\left[\mathbf{G}_{V}\right]^{\bullet q(n)}$.

- $\left.T\right|_{[\tau, t]}$ is holonomically isolated

This follows from Proposition 6.3. because

and $\left[\mathbf{G}_{V}\right]^{\bullet q(n)}$ is a subgroup of $\mathbf{G}^{n}$.

$$
\mathcal{A}_{\left.T\right|_{[\tau, t]}}^{\iota}=\left[\mathbf{G}_{V}\right]^{\bullet q(n)}=\prod_{s=1}^{S q(n)} \mathbf{G}_{V^{(s)}}
$$

- $\mathcal{A}_{\left.T\right|_{[\tau, t]} ^{\iota}}$ is independent of the ultralocal trivialization

Let now $\iota^{\prime}$ be an arbitrary ultralocal trivialization. Then $\iota$ and $\iota^{\prime}$ are related by a generalized gauge transform ${ }^{6}$. Consequently, we have

$$
\mathcal{A}_{\left.T\right|_{[\tau, t]} ^{\prime}}^{\prime^{\prime}}=\left(\vec{g}_{T(\tau)}\right)^{-1} \mathcal{A}_{\left.T\right|_{[\tau, t]}}^{\iota} \vec{g}_{T(t)}
$$

for some $\vec{g}_{T(\tau)}, \vec{g}_{T(t)} \in \mathbf{G}^{n}$. Since gauge transformations depend only on the endpoints of paths, equal endpoints lead to equal components in these two elements of $\mathbf{G}^{n}$. Hence, we have $\vec{g}_{T(\tau)} \in \mathbf{G}_{V(T(\tau))}$ and $\vec{g}_{T(t)} \in \mathbf{G}_{V(T(t))}$.

By Proposition 3.5 we get $V(T(\tau)) \leq V\left(T\left(\tau^{\prime}\right)\right)$ and $V(T(t)) \leq V\left(T\left(t^{\prime \prime}\right)\right)$ for some $T$-regular $\tau^{\prime}, t^{\prime \prime} \in[\tau, t]$. Thus, $\mathbf{G}_{V(T(\tau))} \subseteq \mathbf{G}_{V\left(T\left(\tau^{\prime}\right)\right)}$ and $\mathbf{G}_{V(T(t))} \subseteq \mathbf{G}_{V\left(T\left(t^{\prime \prime}\right)\right)}$, whence $\mathbf{G}_{V(T(\tau))}$ and $\mathbf{G}_{V(T(t))}$ are contained in $\mathbf{G}_{V}$. Since $\left[\mathbf{G}_{V}\right]^{\bullet q(n)}$ is a group, we have

$$
\mathcal{A}_{\left.T\right|_{[\tau, t]}}^{\iota^{\prime}}=\left(\vec{g}_{T(\tau)}\right)^{-1} \mathcal{A}_{\left.T\right|_{[\tau, t]}}^{\iota} \vec{g}_{T(t)}=\left(\vec{g}_{T(\tau)}\right)^{-1}\left[\mathbf{G}_{V}\right]^{\bullet q(n)} \vec{g}_{T(t)}=\left[\mathbf{G}_{V}\right]^{\bullet q(n)} .
$$

Corollary 7.6 Let $\mathbf{G}$ be as in Theorem [7.5. Then for every web $w$ and every $t \in(0,1]$ there is some $t^{\prime} \in(0, t]$, such that for every $0 \leq \tau \leq t^{\prime}$

- $\left.w\right|_{[\tau, t]}$ is holonomically isolated;

- $\mathcal{A}_{\left.w\right|_{[\tau, t]}}$ is a Lie subgroup of $\mathbf{G}^{n}$ with

$$
\mathcal{A}_{\left.w\right|_{[\tau, t]}}=\mathrm{X}_{i} \mathcal{A}_{\left.T_{i}\right|_{[\tau, t]}}=\mathrm{X}_{i}\left[\mathbf{G}_{V_{T_{i}}}\right] \bullet q\left(\# T_{i}\right)
$$

independent of the chosen ultralocal trivialization.

\footnotetext{
${ }^{6}$ A generalized gauge transform is a function from $M$ to $\mathbf{G}$.
} 
Here, $w=T_{1} \cup \ldots \cup T_{W}$ is a decomposition of $w$ into tassels $T_{i}$.

Proof This is an immediate consequence of the Theorem above and Lemma 5.2 since two tassels share at most the endpoints of their paths with parameter value $1 . \quad$ qed

Setting $t$ to 1 and $\tau$ to 0 , we get with the same notations

Corollary 7.7 Let $\mathbf{G}$ be as in Theorem 7.5. Then every web $w$ is holonomically isolated with $\mathcal{A}_{w}=\mathrm{X}_{i}\left[\mathbf{G}_{V_{w}}\right]^{\bullet q(\# w)}$ being a Lie subgroup of $\mathbf{G}^{\# w}$. This again is independent of the chosen trivialization.

Proof Every $v \in V_{i}:=V_{T_{i}}$ can be interpreted as some $\widehat{v} \in V_{w}$ simply by adding zeros at all components that do not correspond to paths in $T_{i}$. Since the only intersection points for tassels are at parameter value 1 being not $w$-regular, each $\widehat{v} \in V_{w}$, on the other hand, corresponds to precisely one $i$ and one $v \in V_{i}$. We have now

$$
\begin{aligned}
\mathbf{G}_{\widehat{v}}=\left\{\left(e_{\mathbf{G}}, \ldots, e_{\mathbf{G}}\right)\right\} & \times \ldots \times\left\{\left(e_{\mathbf{G}}, \ldots, e_{\mathbf{G}}\right)\right\} \times \mathbf{G}_{v} \times \\
& \times\left\{\left(e_{\mathbf{G}}, \ldots, e_{\mathbf{G}}\right)\right\} \times \ldots \times\left\{\left(e_{\mathbf{G}}, \ldots, e_{\mathbf{G}}\right)\right\}
\end{aligned}
$$

with $\mathbf{G}_{v}$ at component $i$. Consequently, giving $V_{w}$ the ordering induced by the sequence of orderings in $V_{1}, \ldots, V_{n}$, we have $\times_{i} \mathbf{G}_{V_{i}}=\mathbf{G}_{V_{w}}$. Moreover, since elements of $V_{w}$ lead to commuting $\mathbf{G}_{\widehat{v}}$ s, if they correspond to different tassels, we have $\mathbf{X}_{i}\left[\mathbf{G}_{V_{i}}\right]^{\bullet q}=\left[\mathbf{G}_{V_{w}}\right]^{\bullet q}$ for all $q \in \mathbb{N}$. By $\# w \geq \# T_{i}$ for all $i$, we get $q(\# w) \geq q\left(\# T_{i}\right)$ and thus

$$
\mathcal{A}_{\left.w\right|_{[\tau, t]}}=\mathbf{X}_{i}\left[\mathbf{G}_{V_{i}}\right]^{\bullet q\left(\# T_{i}\right)}=\mathbf{X}_{i}\left[\mathbf{G}_{V_{i}}\right]^{\bullet q(\# w)}=\left[\mathbf{G}_{V_{w}}\right]^{\bullet q(\# w)}
$$

by the group property of each $\left[\mathbf{G}_{V_{i}}\right]^{\bullet q\left(\# T_{i}\right)}$.

qed

Using Theorem 4.1 we get

Corollary 7.8 Let $\mathbf{G}$ be as in Theorem 7.5

If $\mathbf{G}$ is semisimple, then every web is strongly holonomically independent.

These two corollaries yield, moreover, a new proof for the denseness results of smooth connections as a subset of generalized connections, differing from that presented in 8 ] and now including also a huge class of non-compact structure groups:

Proposition 7.9 Assume $\mathbf{G}$ as in Theorem 7.5 and let $\operatorname{dim} M \geq 2$.

Then, in the category of piecewise smooth and immersive paths, $\mathcal{A}$ is dense in $\overline{\mathcal{A}}$ iff $\mathbf{G}$ is semisimple.

Here, $\overline{\mathcal{A}}=\operatorname{Hom}(\mathcal{P}, \mathbf{G})$ is the set of all generalized connections [1, 6]. Note that this definition is (for non-semisimple $\mathbf{G}$ ) different from the definition by $\lim _{w} \mathcal{A}_{w}$ in [3] that uses $\mathcal{A}_{w}$ instead of $\overline{\mathcal{A}}_{w}=\mathbf{G}^{\# w}$.

Proof Recall [8] that $\mathcal{A}$ is dense in $\overline{\mathcal{A}}$ if $\mathcal{A}_{w}=\mathbf{G}^{\# w}$ for all webs $w$. This is given for connected semisimple $\mathbf{G}$ according to Corollary [7.8. On the other hand, recall that $\mathcal{A}$ is not dense in $\overline{\mathcal{A}}$ if $\mathcal{A}_{w}$ is not dense in $\mathbf{G}^{\# w}$ for some web $w$. However, by Proposition 4.11 this is the case if $\mathbf{G}$ is not semisimple: Observe first that there is always a web having type $V$ with $\operatorname{dim} \operatorname{span}_{\mathbb{R}} V<\# w$ (e.g., the web given in [2]; or see Figure 1 on page 13) and second that a Lie subgroup of non-zero codimension is never a dense subgroup.

qed 
Finally, we remark that Corollary 7.7 above completes a purely algebraic verification of the statement by Baez and Sawin in [3] that for connected and compact groups the parallel transports along a tassel form a Lie subgroup of $\mathbf{G}^{\# T}$. Here, however, the assumptions have been significantly weakened: $\mathbf{G}$ can now be any connected reductive linear algebraic group. This includes, in particular, all classical semisimple Lie groups - be they compact or not. Therefore, our results may be extended also to, e.g., $\mathbf{G}=S l(2, \mathbb{C})$ being relevant for the non-compactified version of loop quantum gravity. Already in the compact case, the assumption of the Lie subgroup property was crucial to initiate a theory of well-defined generalized measures 3]. Although a measure theory in the non-compact case is still more or less on a speculative level, our results together with those in [5] may give some hope that, after these problems are solved for piecewise analytic paths, the measure theory can even be extended to the smooth category.

\section{Acknowledgements}

I am very grateful to Jerzy Lewandowski, Andrzej Okołów and Matthias Schmidt for fruitful discussions. Moreover, I thank John Baez and Stephen Sawin for clarifying some point in their paper 3]. Finally, I am very grateful that this work has been supported by the ReimarLüst-Stipendium of the Max-Planck-Gesellschaft and in part by NSF grant PHY-0090091.

\section{Appendix}

\section{A Holonomy Independence in the General Case}

Proposition A.1 Let $\mathbf{G}$ be some connected Lie group and let $\gamma=\left\{\gamma_{1}, \ldots, \gamma_{n}\right\}$ be some finite set of non-selfintersecting paths in the manifold $M$. Suppose that for every $\gamma_{i} \in \gamma$ there is a closed interval $I_{i} \subseteq[0,1]$ and some open neighbourhood $U_{i}$ of $\gamma_{i}\left(I_{i}\right)$ such that im $\gamma_{i} \cap U_{i} \hookrightarrow U_{i}$ is an embedding. Assume that all $U_{i}$ can be chosen mutually disjoint.

Then for every ultralocal trivialization $\iota$, for every $A \in \mathcal{A}$ and for all $g_{1}, \ldots, g_{n} \in \mathbf{G}$ there is some $A^{\prime} \in \mathcal{A}$ such that

- $h_{\gamma_{i}}^{\iota}\left(A^{\prime}\right)=g_{i}$ for all $i$;

- $A$ and $A^{\prime}$ coincide outside $\bigcup_{n} U_{n}$.

Proof Observe first that by induction we may assume $n=1$. Moreover, if the statement of the proposition is true for one trivialization, it is true for every trivialization. By the local triviality of the principal fibre bundle $P(M, G)$, we may assume that $P$ is trivial over $U:=U_{1}$ and that $\gamma$ is smooth on $I:=I_{1}$ (otherwise, shrink $U$ and $I$, if necessary). Hence, we may fix some ultralocal trivialization $\iota$ being smooth on $U$. In the following, however, we will simply write $h_{A}$ instead of $h_{A}^{\iota}$.

We now proceed in two steps. First we modify $A$ such that it becomes "zero" in $\gamma(I)$ and second we modify it there to get the desired parallel transports.

- First, since $M$ is a manifold, there is some smooth function $f$ on $M$ being 1 on $\gamma(I)$ with supp $f \subseteq U$. We define $A_{f}$ to be equal $(1-f) A$ on $U$ w.r.t. $\iota$ and equal $A$ outside. It is clear that $A_{f}$ is again a smooth connection. To furnish the first part, we define $g^{\prime}:=h_{A_{f}}\left(\left[0, \tau^{-}\right]\right)^{-1} g h_{A_{f}}\left(\left[\tau^{+}, 1\right]\right)^{-1}$.

- Recall that $g^{\prime}$ - as every element in a connected Lie group $\mathbf{G}$ - can be written as a product $g_{1} \cdots g_{l}$ of finitely many $g_{j} \in \exp (\mathfrak{g})$, where $\mathfrak{g}$ denotes the Lie 
algebra to $\mathbf{G}$, i.e., $g_{j}=\exp \left(B_{j}\right)$ with $B_{j} \in \mathfrak{g}$. Divide now $I$ into $2 l-1$ intervals $I_{1}, I_{1 \frac{1}{2}}, I_{2}, \ldots, I_{l}$ by inserting $2 l-2$ points and choose for all integer $j$ some open neighbourhood $V_{j} \subseteq U$ of some interior point in $I_{j}$, such that all these neighbourhoods are mutually disjoint and $V_{j} \cap \operatorname{im} \gamma \subseteq \gamma\left(I_{j}\right)$. Choose finally (again for integer $j$ ) some smooth sections $f_{j}: M \longrightarrow T^{*} M$ with supp $f_{j} \subseteq V_{j}$ and fulfilling $\int_{I_{j}} f_{j}(\gamma(t))_{\mu} \dot{\gamma}^{\mu}(t) \mathrm{d} t=1$. Now we define $A^{\prime}$ to be equal $A_{f}+\sum_{j} f_{j} B_{j}$ on $\bigcup_{j} V_{j} \subseteq U$ and equal $A_{f}$ outside. It is clear that $A^{\prime}$ is again a smooth connection coinciding with $A$ outside $U$. Since $A^{\prime}$ equals $f_{j} B_{j}$ on $V_{j}$ and since $B_{j}$ is a constant, we have for integer $j$

and thus

$$
h_{A^{\prime}}\left(\left.\gamma\right|_{I_{j}}\right)=\exp \left(\int_{I_{j}} f_{j}(\gamma(t))_{\mu} B_{j} \dot{\gamma}^{\mu}(t) \mathrm{d} t\right)=\exp \left(B_{j}\right)=g_{j}
$$

$$
\begin{aligned}
h_{A^{\prime}}(\gamma) & =h_{A^{\prime}}\left(\left.\gamma\right|_{\left[0, \tau^{-}\right]}\right)\left(\prod_{j=1,2 j \in \mathbb{N}}^{l} h_{A^{\prime}}\left(\left.\gamma\right|_{I_{j}}\right)\right) h_{A^{\prime}}\left(\left.\gamma\right|_{\left[\tau^{+}, 1\right]}\right) \\
& =h_{A_{f}}\left(\left.\gamma\right|_{\left[0, \tau^{-}\right]}\right)\left(\prod_{j=1}^{l} g_{j}\right) h_{A_{f}}\left(\left.\gamma\right|_{\left[\tau^{+}, 1\right]}\right) \\
& =h_{A_{f}}\left(\left.\gamma\right|_{\left[0, \tau^{-}\right]}\right) g^{\prime} h_{A_{f}}\left(\left.\gamma\right|_{\left[\tau^{+}, 1\right]}\right) \\
& =g .
\end{aligned}
$$

qed

\section{References}

[1] Abhay Ashtekar and Jerzy Lewandowski: Projective techniques and functional integration for gauge theories. J. Math. Phys. 36 (1995) 2170-2191. e-print: gr-qc/9411046.

[2] John C. Baez and Stephen Sawin: Diffeomorphism-invariant spin network states. J. Funct. Anal. 158 (1998) 253-266. e-print: q-alg/9708005.

[3] John C. Baez and Stephen Sawin: Functional integration on spaces of connections. J. Funct. Anal. 150 (1997) 1-26. e-print: q-alg/9507023

[4] Armand Borel: Class Functions, Conjugacy Classes and Commutators in Semisimple Lie Groups. In: Algebraic Groups and Lie Groups, edited by Gus Lehrer, A. L. Carey, J. B. Carrell, M. K. Murray, and T. A. Springer, pp. 1-19, Austral. Math. Soc. Lect. Ser. 9 (Cambridge University Press, Cambridge, 1997).

[5] Christian Fleischhack: Hyphs and the Ashtekar-Lewandowski Measure. J. Geom. Phys. 45 (2003) 231-251. e-print: math-ph/0001007.

[6] Christian Fleischhack: Mathematische und physikalische Aspekte verallgemeinerter Eichfeldtheorien im Ashtekarprogramm (Dissertation). Universität Leipzig, 2001.

[7] Christian Fleischhack: Proof of a Conjecture by Lewandowski and Thiemann. MIS-Preprint 32/2003, CGPG-03/3-7. e-print: math-ph/0304002

[8] Christian Fleischhack: Regular Connections among Generalized Connections. J. Geom. Phys. (to appear). MIS-Preprint 101/2002, CGPG-02/11-03. e-print: math-ph/0211060.

[9] Christian Fleischhack: Stratification of the Generalized Gauge Orbit Space. Commun. Math. Phys. 214 (2000) 607-649. e-print: math-ph/0001006 math-ph/0001008

[10] Jerzy Lewandowski and Thomas Thiemann: Diffeomorphism invariant quantum field theories of connections in terms of webs. Class. Quant. Grav. 16 (1999) 2299-2322. e-print: gr-qc/9901015. 
[11] A. L. Onishchik (ed.): Lie Groups and Lie Algebras III (Encyclopaedia of Mathematical Sciences 41). Springer-Verlag, Berlin, 1994. 